\title{
Combined length scale and overhang angle control in minimum compliance topology optimization for additive manufacturing
}

\author{
Jeroen Pellens · Geert Lombaert • Boyan Lazarov • Mattias Schevenels
}

Received: date / Accepted: date

\begin{abstract}
This paper focusses on topology optimization for additive manufacturing. In order to ensure that the optimized design is immediately manufacturable, it is essential to take into account the appropriate geometric constraints during the optimization. Two important constraints are minimum length scale and maximum overhang angle. A minimum length scale is needed to ensure that the condition on minimal printable feature sizes is satisfied, while an imposed overhang angle eliminates the need for a temporary support structure. This paper first shows that both constraints cannot simultaneously be met by a straightforward coupling of existing methods for length scale and overhang angle control. Next, a new filtering scheme is introduced, based on a specific combination of spatial filters, which allows direct control over these constraints in a minimum compliance topology optimization problem. A 2D
\end{abstract}

\footnotetext{
J. Pellens

Department of Architecture, Faculty of Engineering Science, KU Leuven, Kasteelpark Arenberg 1

3001 Leuven, Belgium

E-mail: jeroen.pellens@kuleuven.be

\section{G. Lombaert}

Department of Civil Engineering, Faculty of Engineering Science, KU Leuven, Kasteelpark Arenberg 40

3001 Leuven, Belgium

B. Lazarov

School of Mechanical, Aerospace and Civil Engineering, The University of Manchester, Manchester

M13 9PL, UK

M. Schevenels

Department of Architecture, Faculty of Engineering Science, KU Leuven, Kasteelpark Arenberg 1

3001 Leuven, Belgium
}

benchmark problem and a complex 3D case study are presented to demonstrate that the proposed filtering scheme successfully imposes a target length scale in both the solid and the void phase of the design domain, while simultaneously allowing control over the overhang angle.

Keywords Topology optimization - Additive manufacturing - Length scale control - Overhang angle control $\cdot$ Manufacturing constraints

\section{Introduction}

Topology optimization is a numerical, iterative method for finding the optimal distribution of material inside a specified design domain [1-3]. In the density based approach, the design domain is represented using a density field which takes value 1 at elements where material is present, and 0 in the void regions. Intermediate densities are also allowed to facilitate the use of a gradient-based optimization scheme, but they are penalized in order to obtain a black-and-white solution. The design's geometry is usually controlled with the help of filters. Their function is to avoid checkerboard patterns [4] and ensure mesh independent solutions [5]. Most filters consist of a spatial averaging operation where the density of an element is replaced by the weighted average of its neighboring elements $[6,7]$. Such an operation imposes a minimum length scale on the solid and void phases in the design domain. These solutions, by definition, possess a significant part of intermediate densities which have no direct physical counterpart. A Heaviside projection $[8,9]$ is used to guide the design to a black-and-white solution and to ensure manufacturability. However, direct length scale control on both phases is lost in 
this process. An alternative filtering strategy proposed in [10] combines morphological operators originally developed for image processing [11] and imposes a target minimum length scale in one of the phases while ensuring black-and-white solutions. Wang et al. [12] suggest to use the so-called robust formulation to ensure control over the length scale in both the solid and void phase simultaneously. The robust formulation is computationally expensive as the problem has to be solved for multiple geometries. For minimum compliance problems, Lazarov et al. [13] showed that only a single geometry must be considered, so reducing the computational cost to the cost of the original (non-robust) problem. A recent study by Wadbro and Hägg [14] uses morphological operators to form an alternative filtering scheme resulting in direct control over the length scale in both solid and void phase. Strategies for imposing a minimum length scale using the level set approach and the moving morphable components approach are proposed by Allaire et al. [15] and Zhang et al. [16], respectively.

Topology optimization usually results in very structurally efficient, but complicated shapes that are hard and expensive to produce using traditional techniques. Additive manufacturing ( $\mathrm{AM}$ ), a relatively new and rapidly evolving production technique, eliminates many of these limitations by building components in a layerby-layer fashion using computer guided machines. The technique allows for a high degree of design freedom and is capable of producing sophisticated geometries at only a fraction of the costs for traditional manufacturing [17]. Although AM techniques were initially only viable for rapid prototyping with polymers, recent improvements, especially in metal-based techniques, extend their application to end-usable parts [18].

Up to now AM has often been used for the production of elements designed for traditional manufacturing. This is far from cost-effective, and a better efficiency can be obtained by redesigning these elements to exploit all advantages of AM [19,20]. Additive manufacturing is still subjected to a couple of critical technological constraints, however. The minimum printable feature size has to be taken into account and an element often needs a temporary support structure to remain stable during the production. Printing and removing support structures is a time and material consuming process, which decreases the overall efficiency. Thus, recent research heavily focuses on eliminating the need for temporary support structures [21-25] by imposing a maximum overhang angle. This angle is defined as the maximum allowable angle between a downward facing element and the building direction (direction in which the layers are deposited) for which the element is self-supporting, and is typically between $40^{\circ}$ and $50^{\circ}$ [26,27].

Gaynor and Guest [23] proposed a method for overhang angle control by filtering using a wedge-shaped support. A different approach is described by Qian et al. [24], where the level curves of the design are used to identify the violation of the required overhang angles. Both methods are based on an additional constraint which is formulated in terms of a smoothened approximation of a $\min / \max$ operator. The error introduced by this approximation is exploited by the optimization algorithm, resulting in sawtooth patterns with gradually decreasing densities underneath overhanging surfaces. To avoid the problems occurring in these strict support elimination methods, Mirzendehdel and Suresh [28] developed an optimization framework based on the level set approach in which a design's performance is optimized within a maximum support structure constraint.

A recent study by Langelaar [25, 29] proposed a different approach to the overhang angle constraint problem. The method uses a spatial filtering technique to transform a blueprint design to a printable design in which every element is sufficiently supported. In a discrete case, where all elements are black or white, this filter checks the element densities row by row, starting at the baseplate, and gradually deletes all elements which are not supported by elements in the previous row. This technique results in fast and stable convergence to a printable design and avoids the aforementioned sawtooth problem by using a filter instead of adding a constraint function.

Alternative strategies aim at simulating the layerby-layer printing process in the formulation of the optimization problem. Allaire et al. [30,31] employs this technique in shape optimization problems, while Amir and Mass [32] propose a method for incorporating the construction process in topology optimization problems. Although the same sawtooth patterns appear in some shape optimization cases, general results of both strategies show an important decrease in the amount of support structure needed.

In this paper, the strategies for length scale control by Wadbro and Hägg [14] and overhang angle control by Langelaar [25] are combined to form a new filtering scheme guaranteeing direct control over both constraints simultaneously in the final design. Intuitively, a simple sequential coupling of both strategies is expected to provide the required length scale and over- 
hang angle control. However, the effect introduced by the first filter, length scale or overhang angle control, will be neutralized by the second filter, which results in designs with no length scale or overhang angle control. Based on these results, a new, more complex filtering scheme is presented which proves capable of correctly imposing both constraints simultaneously. The new filtering scheme is composed of several Heaviside filters combined with the overhang angle control filter proposed by Langelaar [25]. As a side benefit, the filtering scheme introduces a controllable fillet radius for the interior corners of the final design.

This paper starts with a brief recapitulation of the minimum compliance topology optimization strategy (section 2). Next, the manufacturability filters used to control the length scale on both phases of the design and the maximum overhang angle are described (section 3). Next, two straightforward combination strategies are presented and tested and a new filtering scheme is introduced and validated (section 4). Following the 2D validation of the method, a $3 \mathrm{D}$ case study is described (section 5). Finally, conclusions are provided. The sensitivity analyses of the described filtering strategies are added as an appendix to this paper.

\section{Minimum compliance topology optimization}

\subsection{Formulation of the optimization problem}

In topology optimization, the design domain is divided in a finite number of elements. Each finite element $e$ is given a density $\rho_{e}$ (zero density for void, unit density for solid elements) to determine the amount of material. The minimum compliance optimization problem considered in this study is formulated as:

$$
\begin{array}{cl}
\min _{\boldsymbol{\rho}} & c(\boldsymbol{\rho})=\mathbf{f}^{T} \mathbf{u}(\boldsymbol{\rho}) \\
\text { s.t. } & \mathbf{K}(\boldsymbol{\rho}) \mathbf{u}(\boldsymbol{\rho})=\mathbf{f} \\
& V(\boldsymbol{\rho}) \leq V^{*} \\
& \mathbf{0} \leq \boldsymbol{\rho} \leq \mathbf{1},
\end{array}
$$

where $\mathbf{u}$ and $\mathbf{f}$ are the displacement and force vectors, respectively. They are related by the equilibrium equation $\mathbf{K u}=\mathbf{f}$, where $\mathbf{K}$ is the stiffness matrix obtained by the finite element method. The set of constraints includes box constraints on the design variables, stating that $\rho_{e}$ can only take a value between 0 and 1 , and a volume constraint, stating that the total volume $V$ of the design must be smaller than the maximum allowed volume $V^{*}$. The volume $V$ is calculated as:

$V(\boldsymbol{\rho})=\frac{\sum_{e=1}^{N} \rho_{e} v_{e}}{\sum_{e=1}^{N} v_{e}}$, where $v_{e}$ represents the volume of element $e$ and $N$ the total number of elements in the design domain.

This study uses the widely accepted Solid Isotropic Material with Penalization (SIMP) approach to topology optimization [33]. In order to obtain a continuous optimization problem, all densities ranging from zero to one are allowed, but intermediate densities are penalized as they are not desirable in the final design. A nonlinear relation between an element's density $\rho_{e}$ and its corresponding stiffness $E_{e}$ is used for the penalization:

$E_{e}\left(\rho_{e}\right)=E_{\min }+\rho_{e}^{p}\left(E_{0}-E_{\min }\right)$,

where $E_{0}$ and $E_{\min }$ represent the stiffness of the solid phase and the void phase of the design, respectively, and $p>1$ is the penalization factor used to ensure black-and-white solutions, typically $p=3$. By using the element densities $\rho_{e}$ as design variables, the optimization process will lead to mesh dependent solutions and checkerboard patterns [4,34]. These problems can be avoided by applying a density filter to the design variables $[6,7]$. This spatial filter replaces the density of an element $\rho_{e}$ by the weighted average $\tilde{\rho}_{e}$ of its neighboring elements, and is defined as:

$\tilde{\rho}_{e}=\frac{\sum_{j \in \mathbb{N}_{e}} h_{e j}^{R} \rho_{j}}{\sum_{j \in \mathbb{N}_{e}} h_{e j}^{R}}$,

where $h_{e j}^{R}$ is the filter kernel with radius $R$ defined as:

$h_{e j}^{R}=\max \left(R-\left\|x_{e}-x_{j}\right\|, 0\right)$.

where $\left\|x_{e}-x_{j}\right\|$ is the center-to-center distance from element $e$ to $j$. In equation (4) $\mathbb{N}_{e}$ is the neighborhood set, or the set of all elements $j$ for which the distance $\left\|x_{e}-x_{j}\right\|$ is smaller than or equal to the filter radius $R$.

The density filter leads to a design with a significant number of intermediate densities which have no direct physical counterpart. To avoid this, a Heaviside projection is applied to the filtered design allowing for a strict black-and-white solution $[8,9]$. This projection technique transforms the filtered densities $\tilde{\rho}_{e}$ to physical densities $\bar{\rho}_{e}$ using a Heaviside step function, meaning that all values smaller than a specific threshold $\eta$ are projected to 0 and all densities larger than $\eta$ to 1. Here, the smooth approximation proposed in [12] is adopted to ensure differentiability:

$\bar{\rho}_{e}=\frac{\tanh (\beta \eta)+\tanh \left(\beta\left(\tilde{\rho}_{e}-\eta\right)\right)}{\tanh (\beta \eta)+\tanh (\beta(1-\eta))}$

The Heaviside parameter $\beta$ is used to control the smoothness of the approximation: for $\beta$ approaching 
zero, the filter tends to a linear projection, while for $\beta$ approaching infinity, it corresponds to the exact Heaviside step function. A continuation scheme is followed for the Heaviside parameter $\beta$ in order to ensure smooth convergence of the optimization: in the first iteration, a low value for $\beta$ is used, and in every subsequent iteration, the value $\beta$ is multiplied with a factor slightly greater than 1 , until it becomes larger than or equal to $\beta_{\max }$. In this paper, the combination of a density filter and a Heaviside projection is referred to as the Heaviside filter.

As every method described in this paper uses a different filtering strategy, a schematic representation of each strategy used is included in order to retain an overview. The filtering strategy used in this section proceeds as follows:<smiles>[2H]P([18O])[18OH]</smiles>

The design variables $\rho$ in this optimization process are transformed to element densities $\overline{\boldsymbol{\rho}}$ by using the previously described Heaviside filter with a threshold $\eta$ equal to 0.5 . Both the compliance and volume are calculated using these filtered element densities.

\subsection{Test example}

In this subsection, the previously described filtering method is demonstrated using a $2 \mathrm{D}$ benchmark problem. This problem is used through the rest of the paper and is therefore only described in this subsection.

The 2D benchmark case, presented in fig. 1, is a minimum compliance problem with a volume constraint and consists of the design of a cantilever beam with length $L$ and height $\frac{L}{2}$. The left side of the domain is clamped and a unit load is applied to the center of the right side of the beam. The design domain is discretized using $n=200 \times 100=20000$ square $\mathbb{Q}_{1}$ finite elements with unit dimensions. A Young's modulus $E_{0}=1$ for the solid phase and $E_{\min }=10^{-9}$ for the void phase is used, and Poisson's ratio $\nu$ is set to 0.3 . The volume fraction is set to $45 \%$ of the total volume of the design domain. A filter radius $R$ of $0.015 \mathrm{~L}$ is used. SIMP interpolation is adopted with penalization power $p=1$ going to $p_{\max }=3$ using a continuation scheme, where $p$ increases with a factor 1.01 in every iteration. A similar continuation scheme is applied to the Heaviside parameter $\beta$, starting at $\beta_{0}=1$ and increasing by a factor 1.01 in every iteration until $\beta \geq 30$. The optimization is performed using the Method of Moving Asymptotes (MMA) [35]. Convergence is assumed if the maximum change in element densities between iterations is less than 0.008 . All computations are performed on a MacBook Pro 2015 with a $2.7 \mathrm{GHz}$ Intel Core i5 processor.

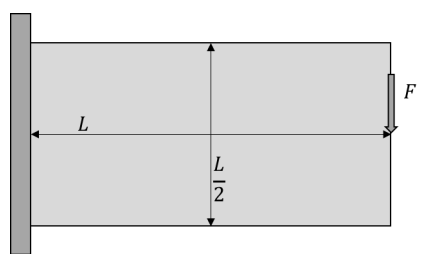

Fig. 1: Design domain and boundary conditions for the cantilever beam.

The design obtained as the solution of optimization problem (1) is shown in figure 2. The compliance is equal to $c=1627$.

In order to assess the degree to which a blackand-white design is obtained, the measure of nondiscreteness $M_{\text {nd }}$ is calculated [10]:

$M_{\mathrm{nd}}=\frac{\sum_{e=1}^{N} 4 \bar{\rho}_{e}\left(1-\bar{\rho}_{e}\right)}{N} \times 100 \%$

For $M_{\text {nd }}=0 \%$ the design is perfectly discrete, while $M_{\mathrm{nd}}=100 \%$ corresponds to a solution where all elements have intermediate densities [10]. For this example, the measure of non-discreteness is $M_{\mathrm{nd}}=2.50 \%$.

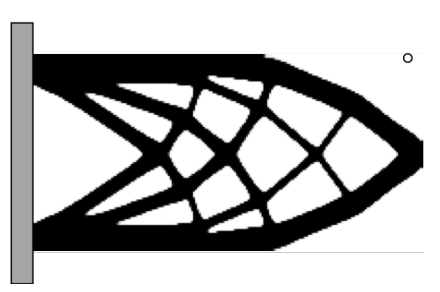

Fig. 2: Design optimized without length scale or overhang angle control, $c=1627, M_{\text {nd }}=2.50 \%$. The circle indicates the filter radius $R$. 


\section{Manufacturability filters}

\subsection{Length scale filter}

This subsection describes a strategy based on the approach by Wadbro and Hägg [14] to provide length scale control in the solid and the void phase of the design. It is based on the sequential application of the Heaviside formulation [37] of the dilate, erode, open and close filters described by Sigmund [10]. If the threshold $\eta$ is set to 0 in the Heaviside projection, all densities $\rho_{e}$ larger than 0 are projected to the solid phase, resulting in a dilated design $\hat{\rho}_{e}$. The dilate filter is therefore given by:

$$
\hat{\rho}_{e}=\frac{\tanh \left(\beta \eta_{\mathrm{d}}\right)+\tanh \left(\beta\left(\frac{\sum_{j \in \mathbb{N}_{e}} h_{e j}^{R} \rho_{j}}{\sum_{j \in \mathbb{N}_{e}} h_{e j}^{R}}-\eta_{\mathrm{d}}\right)\right)}{\tanh \left(\beta \eta_{\mathrm{d}}\right)+\tanh \left(\beta\left(1-\eta_{\mathrm{d}}\right)\right)}
$$

where $\eta_{\mathrm{d}}=0$. If the threshold $\eta$ is set to 1 , all densities $\rho_{e}$ smaller than 1 are projected to the void phase, resulting in an eroded design $\check{\rho}_{e}$. The erode filter is therefore given by:

$$
\check{\rho}_{e}=\frac{\tanh \left(\beta \eta_{\mathrm{e}}\right)+\tanh \left(\beta\left(\frac{\sum_{j \in \mathbb{N}_{e}} h_{e j}^{R} \rho_{j}}{\sum_{j \in \mathbb{N}_{e}} h_{e j}^{R}}-\eta_{\mathrm{e}}\right)\right)}{\tanh \left(\beta \eta_{\mathrm{e}}\right)+\tanh \left(\beta\left(1-\eta_{\mathrm{e}}\right)\right)}
$$

where $\eta_{\mathrm{e}}=1$. Applying an erode filter after a dilate filter performs a close operation on the design. This operation closes all gaps smaller than a specified radius, thus guaranteeing a minimum length scale in the void domain. The resulting closed design is denoted as $\check{\hat{\boldsymbol{\rho}}}$ (note that a piling up of superscripts represents a composition of filters). The inverse of this operation, a dilate filter after an erode filter, results in an open operator. This removes all solid elements smaller than a specified radius, thus guaranteeing a minimum length scale in the solid domain. The resulting opened design is denoted as $\hat{\tilde{\rho}}$. Combining both strategies to obtain open-close/close-open filters has been suggested as a way to achieve a length scale for both solid and void phases of the design [10], but this has been proven false as the second filter neutralizes the effect of the first filter [37].

Wadbro and Hägg [14] proposed an alternative strategy based on the same principles. An open and close filter is applied to the design variables $\boldsymbol{\rho}$, resulting in an opened design $\hat{\tilde{\rho}}$ and a closed design $\check{\hat{\boldsymbol{\rho}}}$. The opened and closed designs are used to calculate the compliance and volume constraint, respectively:<smiles>[Mg]P1NPO1</smiles>

An open filter results in a design with a higher compliance value, as it will never add material, while a close filter results in a design with a higher volume, as it will never remove material. Using the compliance of the opened design and the volume of the closed design, a self penalizing problem is obtained: features that do not satisfy the target length scale contribute to the volume, but not to the compliance, and are therefore avoided by the optimizer.

This approach is applied to the example problem introduced in subsection 2.2. The optimized design is shown in figure 3 . The compliance is equal to $c=1644$ and the measure of non-discreteness is $M_{\mathrm{nd}}=4.50 \%$.

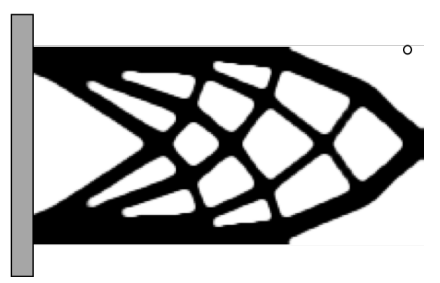

Fig. 3: Design optimized with length scale control; without overhang angle control, $c=1644, M_{\mathrm{nd}}=4.50 \%$.

\subsection{Overhang angle filter}

This subsection describes a spatial filtering technique proposed by Langelaar $[25,29]$ for controlling the maximum overhang angle in the topology optimization process. The basic principles of the method are briefly reviewed in this paper, for more details the reader is referred to the original paper [25].

The overhang angle filter proposed by Langelaar converts a blueprint design $\overline{\boldsymbol{\rho}}$ to a print-ready design $\hat{\boldsymbol{\rho}}$. In the discrete case, where all elements are black or white, this filter starts at the baseplate and goes up one layer at the time, while deleting all elements that are not supported by the previous layer, i.e. all elements for which the entire neighborhood $S_{e}$ (figure 4) is void. In the continuous case, where intermediate densities are allowed, this is implemented as a layer-by-layer filter 
which changes the density of an element $e$ to the minimum of the density of the considered element $e$ and the highest density of the elements in its neighborhood $S_{e}$.

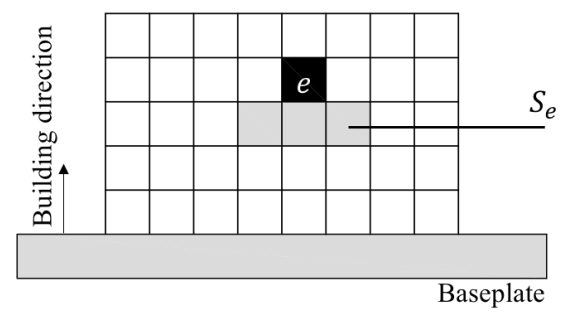

Fig. 4: Definition of the support region $S_{e}$ for an element $e$ in the domain.

A maximum operator is used to determine the highest density of the supporting elements, while a minimum operator determines the modified density for the considered element $e$ :

$\hat{\bar{\rho}}_{e}=\min \left(\bar{\rho}_{e}, \hat{\bar{\rho}}_{e}^{S}\right)$,

where

$\hat{\rho}_{e}^{S}=\max _{i \in S_{e}} \hat{\rho}_{i}$,

and $\hat{\rho}_{i}$ is the density of element $i$ in the support neighborhood $S_{e}$.

Smooth approximations of the min and max operators are introduced to allow for gradient based optimization:

$\hat{\bar{\rho}}_{e}=\frac{1}{2}\left(\bar{\rho}_{e}+\hat{\bar{\rho}}_{e}^{S}-\left(\left(\bar{\rho}_{e}-\hat{\bar{\rho}}_{e}^{S}\right)^{2}+\epsilon\right)^{\frac{1}{2}}+\sqrt{\epsilon}\right)$,

and

$\hat{\bar{\rho}}_{e}^{S}=\left(\sum_{i \in S_{e}} \hat{\bar{\rho}}_{i}^{q}\right)^{1 / q}$,

The parameters $q$ and $\epsilon$ control the accuracy and smoothness of the approximations; for $\epsilon \rightarrow 0$ and $q \rightarrow \infty$ the exact $\min / \max$ operators are obtained. For the examples in this paper the values $q=40$ and $\epsilon=10^{-4}$ are used.

It can be noted that, due to the definition of the overhang filter, the maximum overhang angle is fixed to $45^{\circ}$ in all the examples. According to Langelaar [25], different values for the overhang angle can however be obtained by changing the aspect ratio of the elements.
The overhang angle filtering scheme can be summarized as follows:

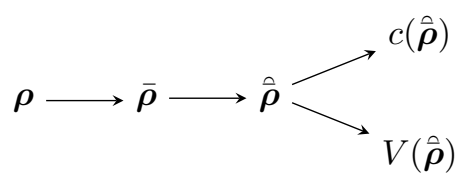

The design variables $\boldsymbol{\rho}$ are projected to discrete densities $\overline{\boldsymbol{\rho}}$, using the Heaviside filter described in section 2.1. The overhang angle filter is applied to these discrete densities, resulting in the physical densities $\hat{\bar{\rho}}$. These physical densities $\hat{\boldsymbol{\rho}}$ are used to calculate the compliance and volume.

The overhang angle filter is applied to the example problem assuming a left-to-right building direction with a maximum allowable overhang angle of $45^{\circ}$. The optimized design with a left-to-right building direction is shown in figure 5 . The compliance is equal to $c=1629$ and the measure of non-discreteness is $M_{\mathrm{nd}}=1.40 \%$.

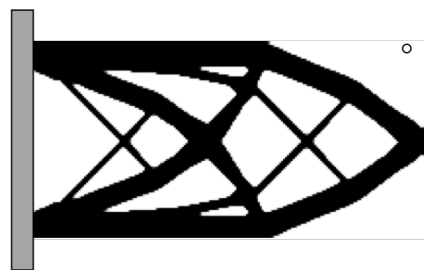

Fig. 5: Design optimized with overhang angle control; without length scale control, $c=1629, M_{\text {nd }}=1.40 \%$.

\subsection{Verification of manufacturability filters}

In order to determine whether the length scale and overhang angle control are effective and to check the manufacturability of the optimized design, various filters are applied to a fully discrete design $\overline{\boldsymbol{\rho}}^{*}$. This design $\overline{\boldsymbol{\rho}}^{*}$ is obtained by projecting the optimized design $\overline{\boldsymbol{\rho}}$ to a strict black-and-white solution, using a projection threshold of 0.5 .

First, an open filter is used to remove solid features that do not satisfy the target length scale, resulting in a design $\hat{\bar{\rho}}^{*}$ with a minimum length scale in the solid phase. The difference between the optimized design and the opened design is quantified as follows:

$\delta_{\mathrm{LS}}=\frac{1}{N} \sum_{e=1}^{N}\left|\bar{\rho}_{e}^{*}-\hat{\bar{\rho}}_{e}^{*}\right|$ 
This difference ratio $\delta_{\mathrm{LS}}$ is a measure for the degree to which the optimized design violates the length scale constraint for the solid phase. It represents the number of elements violating the length scale constraint divided by the total number of elements in the design domain.

Second, a close filter is used in a similar way to remove features in the void phase that do not satisfy the target length scale. The resulting design is denoted as $\check{\hat{\boldsymbol{\rho}}}^{*}$, and the degree $\delta_{\mathrm{LV}}$ to which the length scale constraint in the void phase is violated is determined as follows:

$$
\delta_{\mathrm{LV}}=\frac{1}{N} \sum_{e=1}^{N}\left|\bar{\rho}_{e}^{*}-\check{\hat{\bar{\rho}}}_{e}^{*}\right|
$$

Third, the overhang angle is considered. It should be noted that it is geometrically impossible to simultaneously provide length scale control in the void phase and overhang angle control: as illustrated in figure $6 \mathrm{a}$, the former will lead to rounded interior corners with a radius $R$. At these locations, the overhang angle constraint is violated, as a strict enforcement of the constraint would lead to crisp corners. However, due to the viscosity of the printed material used in additive manufacturing, a 3D printer is capable of manufacturing small bridges as the one shown in figure $6 \mathrm{a}$. In addition, rounded corners are preferred over crisp corners to avoid stress concentration problems [36]. Rounding of interior corners is therefore desirable and should not affect the measure $\delta_{\mathrm{OA}}$ which is used to quantify the degree to which the overhang angle constraint is violated. This measure $\delta_{\mathrm{OA}}$ is therefore determined as follows. First, a dilate filter with radius $R_{\text {void }}$ is applied to the discretized design $\overline{\boldsymbol{\rho}}^{*}$, as illustrated in figure $6 \mathrm{~b}$. This filter does not change the overhang angle, except at the interior corners, where it converts rounded corners to crisp corners. Next, the overhang angle filter proposed by Langelaar $[25,29]$ is used to remove all elements that would need a temporary support structure, as illustrated in figure 6c. Finally, an erode filter is applied to undo the effect of the dilate filter, as illustrated in figure $6 \mathrm{~d}$. The resulting design, illustrated in figure $6 \mathrm{~d}$, is denoted as $\check{\hat{\boldsymbol{\rho}}}^{*}$, and the difference with the original design is quantified as follows:

$\delta_{\mathrm{OA}}=\frac{1}{N} \sum_{e=1}^{N}\left|\bar{\rho}_{e}^{*}-\check{\hat{\hat{\rho}}}_{e}^{*}\right|$

In order to visualize the extent to which an optimized design satisfies the length scale and overhang angle requirements, the fully discrete design $\hat{\rho}^{*}$ is plotted and the elements that violate the requirements

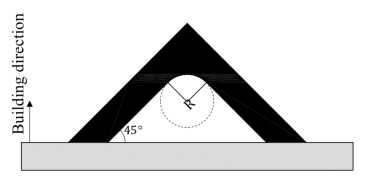

(a) Original design

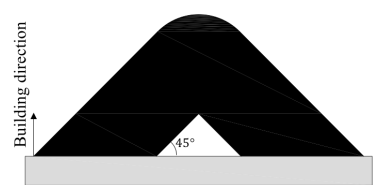

(c) Printable design

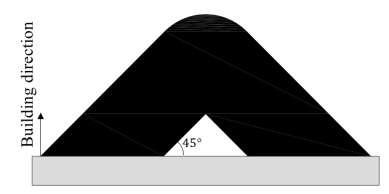

(b) Dilated design

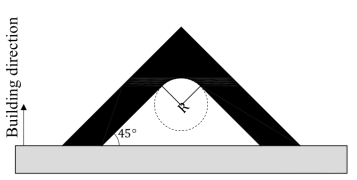

(d) Eroded design
Fig. 6: Schematic representation of the overhang angle check.

(and contribute to the difference ratios $\delta_{\mathrm{LS}}, \delta_{\mathrm{LV}}$, and $\left.\delta_{\mathrm{OA}}\right)$ are shown in red. If a requirement is satisfied, the difference ratio is zero, and no red elements are present.

Figure 7 presents the results of the checks for the design obtained by using the filtering strategy described in subsection 2.1. Figures $7 \mathrm{~b}, 7 \mathrm{c}$ and $7 \mathrm{~d}$ illustrate the calculation of the difference ratios for the optimized design (fig. 7a). The following values are obtained: $\delta_{\mathrm{LV}}=1.00 \%, \delta_{\mathrm{LS}}=2.05 \%$ and $\delta_{\mathrm{OA}}=0.91 \%$ (assuming a left-to-right building direction). These values are non-zero, which means that this strategy does not allow obtaining the target minimum length scale in the solid and void phase of the design. This is due to the Heaviside projection with a threshold $\eta=0.5$, which changes the length scale after it is imposed by the density filter. Although it is possible with the Heaviside filter to impose the correct length scale in the solid phase for $\eta=0$ or in the void phase for $\eta=1$, simultaneous length scale control in both phases is impossible [10]. The value for the difference ratio $\delta_{\mathrm{OA}}$ proves that the design is not self-supporting; which is also illustrated by figure $7 d$. Since the overhang angle was not considered in the optimization process, it is not surprising that a non-zero value is obtained for $\delta_{\mathrm{OA}}$.

Figure 8 presents the results of the checks for the design obtained by using the filtering strategy described in subsection 3.1. The difference ratios $\delta_{\mathrm{LS}}=0.00 \%, \delta_{\mathrm{LV}}=0.00 \%$ and $\delta_{\mathrm{OA}}=0.72 \%$ of the design are calculated as illustrated in figures $8 \mathrm{~b}, 8 \mathrm{c}$ and $8 \mathrm{~d}$. It can be concluded that this strategy correctly imposes the target minimum length scale on the solid and void phase of the design. The non-zero value for the difference ratio $\delta_{\mathrm{OA}}$ proves that the design is not 
self supporting.

Figure 9 presents the results of the checks for the design obtained by using the filtering strategy described in subsection 3.2. The difference ratios $\delta_{\mathrm{LV}}=1.05 \%$, $\delta_{\mathrm{LS}}=3.40 \%$ and $\delta_{\mathrm{OA}}=0.00 \%$ of the design are calculated as illustrated in figures $9 \mathrm{~b}, 9 \mathrm{c}$ and $9 \mathrm{~d}$. It can be concluded that the target length scale is not achieved.

\section{Combined length scale and overhang angle control}

To achieve a filtering scheme providing both length scale and overhang angle control, the strategies described in sections 3.1 and 3.2 should be combined. Two straightforward combinations are tested: applying the overhang angle filter after the length scale filter and applying the length scale filter after the overhang angle filter. Both approaches are described and validated in the following subsection.

\subsection{Strategy 1}

The first filtering strategy used in this section proceeds as follows:

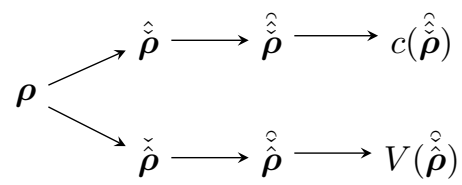

The design variables $\boldsymbol{\rho}$ are transformed to discrete densities $\hat{\tilde{\rho}}$ and $\check{\hat{\boldsymbol{\rho}}}$ by applying an open and close filter to the design, respectively. Next, the overhang angle filter is applied to both designs, resulting in the physical densities $\hat{\hat{\boldsymbol{\rho}}}$ and $\hat{\hat{\boldsymbol{\rho}}}$. The physical densities $\hat{\hat{\boldsymbol{\rho}}}$ acquired by the open filter are used to calculate the compliance, while the physical densities $\hat{\hat{\rho}}$ acquired by the close filter are used to calculate the volume.

The optimized design obtained by this strategy is shown in figure 10a. The compliance is equal to $c=1677$ and the measure of non-discreteness is $M_{\mathrm{nd}}=5.50 \%$. The difference ratios $\delta_{\mathrm{LV}}=0.27 \%$, $\delta_{\mathrm{LS}}=0.57 \%$ and $\delta_{\mathrm{OA}}=0.00 \%$ of the design are calculated as illustrated in figures $10 \mathrm{~b}, 10 \mathrm{c}$ and $10 \mathrm{~d}$. It can be concluded that the target length scale is not achieved in the solid and void phase of the design. This can be explained by the fact that the overhang angle control filter eliminates the effect of the length scale control filter. The zero value for the difference ratio
$\delta_{\mathrm{OA}}$ for a left-to-right building direction proves that the overhang angle control is effective.

\subsection{Strategy 2}

The second filtering strategy used in this section proceeds as follows:

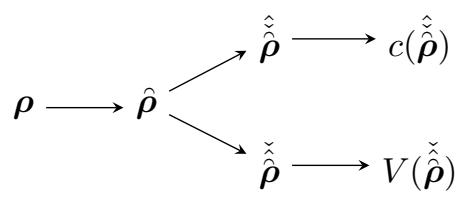

The overhang angle filter is applied to the design variables $\boldsymbol{\rho}$ to form the supported densities $\hat{\boldsymbol{\rho}}$. An open and close filter is applied to the supported densities $\hat{\boldsymbol{\rho}}$, to respectively form the physical densities $\hat{\hat{\boldsymbol{\rho}}}$ and $\check{\hat{\boldsymbol{\rho}}}$. The physical densities $\hat{\hat{\rho}}$ acquired by the open filter are used to calculate the compliance, while the physical densities $\check{\hat{\rho}}$ acquired by the close filter are used to calculate the volume.

The resulting optimized design with a left-to-right building direction is shown in figure 11a. The compliance is equal to $c=1664$ and the measure of nondiscreteness is $M_{\text {nd }}=6.00 \%$. The difference ratios $\delta_{\mathrm{LV}}=0.00 \%, \delta_{\mathrm{LS}}=0.00 \%$ and $\delta_{\mathrm{OA}}=0.00 \%$ of the design are calculated as illustrated in figures $11 \mathrm{~b}, 11 \mathrm{c}$ and $11 \mathrm{~d}$. It can be concluded that this strategy correctly imposes the target minimum length scale on the solid and void phase of the design. The value for the difference ratio $\delta_{\mathrm{OA}}$ for a left-to-right building direction suggests that the overhang angle control is effective. However, if the same problem is optimized using a bottom-to-top building direction, very thin and nonmanufacturable support elements emerge, as shown in figure $12 \mathrm{a}$. The difference ratios $\delta_{\mathrm{LV}}=0.31 \%, \delta_{\mathrm{LS}}=$ $0.56 \%$ and $\delta_{\mathrm{OA}}=9.44 \%$, presented in figures $12 \mathrm{~b}, 12 \mathrm{c}$ and $12 \mathrm{~d}$, demonstrate that the design is not buildable. These thin support elements are not removed by the self-penalizing length scale filter, as their presence has practically no impact on compliance.

The previous examples demonstrate that simultaneous length scale and overhang angle control cannot be achieved by simply combining the existing filters. For this reason, this paper proposes a third, more complex strategy. 


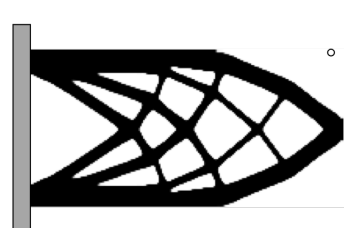

(a) Optimized design; $c=1627, M_{\mathrm{nd}}=2.50 \%$

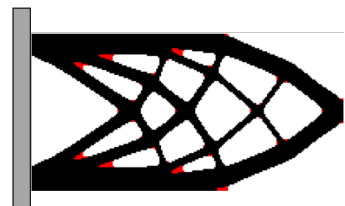

(b) Void length scale check; $\delta_{\mathrm{LV}}=1.00 \%$.

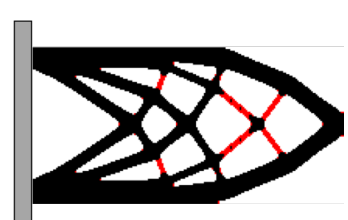

(c) Solid length scale check; $\delta_{\mathrm{LS}}=2.05 \%$.

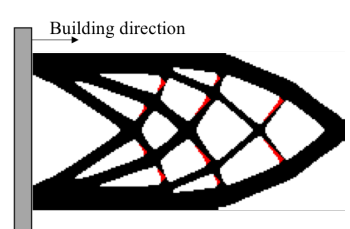

(d) Overhang angle check; $\delta_{\mathrm{OA}}=0.91 \%$.

Fig. 7: Design optimized without length scale or overhang angle control. The circle indicates the filter radius $R$.

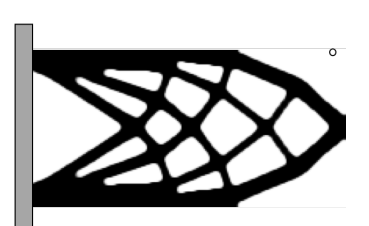

(a) Optimized design; $c=1644, M_{\mathrm{nd}}=4.50 \%$

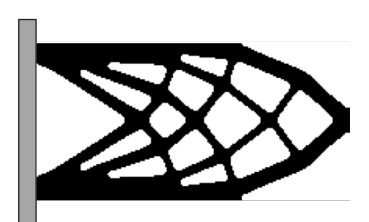

(b) Void length scale check; $\delta_{\mathrm{LV}}=0.00 \%$.

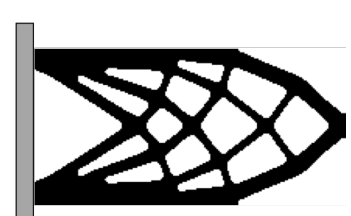

(c) Solid length scale check; $\delta_{\mathrm{LS}}=0.00 \%$.

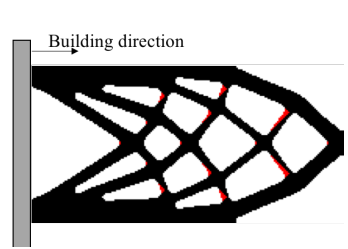

(d) Overhang angle check; $\delta_{\mathrm{OA}}=0.72 \%$.

Fig. 8: Design optimized with length scale control; without overhang angle control.

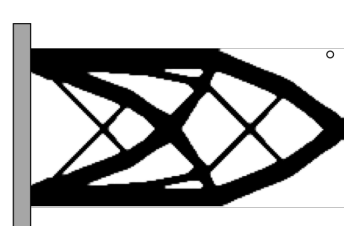

(a) Optimized design; $c=1629, M_{\mathrm{nd}}=1.40 \%$

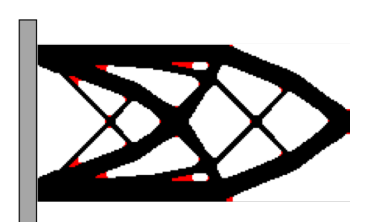

(b) Void length scale check; $\delta_{\mathrm{LV}}=1.05 \%$.

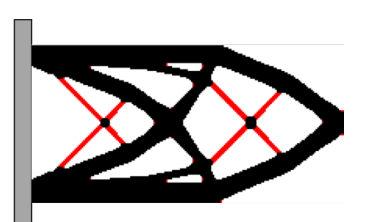

(c) Solid length scale check; $\delta_{\mathrm{LS}}=3.40 \%$.

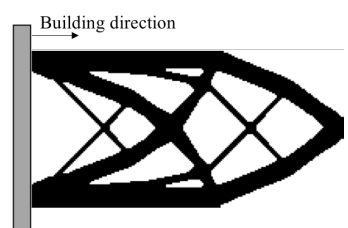

(d) Overhang angle check; $\delta_{\mathrm{OA}}=0.00 \%$.

Fig. 9: Design optimized with overhang angle control; without length scale control.

\subsection{Strategy 3}

The filtering strategy for combined length scale and overhang angle control proposed proceeds as follows:

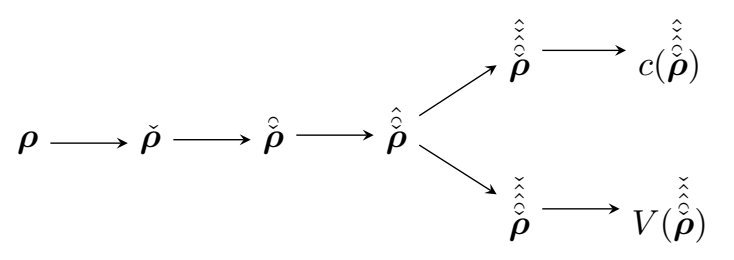

This strategy is based on the strategy proposed in section 4.2 , with two additional filters to avoid the emergence of very thin supporting elements. The main idea is to insert a dilate filter immediately after the overhang angle filter to ensure that all support elements become sufficiently thick. However, the addition of a dilate filter causes the entire filtering sequence to become nonvolume-preserving, leading to non-smooth convergence of the optimization process when the Heaviside parameter $\beta$ is increased. As a solution, an additional erode filter is introduced immediately before the overhang angle filter. This leads to the filtering scheme shown above: first, an erode filter (10) is applied to the design variables $\boldsymbol{\rho}$. Next, the overhang angle filter (12) is applied to the eroded design $\check{\boldsymbol{\rho}}$, resulting in a printable design $\check{\rho}$. To avoid small supporting features, a dilate filter (9) is applied to the design $\tilde{\boldsymbol{\rho}}$, resulting in a dilated design $\hat{\tilde{\rho}}$. Finally, an open/close filter is applied, resulting in an opened design $\hat{\hat{\hat{\rho}}}$, which is used for the compliance calculation, and a closed design $\tilde{\hat{\hat{\rho}}}$, which is used for the volume calculation.

The resulting optimized design for a left-to-right building direction is shown in figure 13a. The compliance is equal to $c=1673$ and the measure of non-discreteness is $M_{\mathrm{nd}}=5.50 \%$. The difference ratios $\delta_{\mathrm{LV}}=0.00 \%, \delta_{\mathrm{LS}}=0.00 \%$ and $\delta_{\mathrm{OA}}=0.00 \%$ of the design are calculated as illustrated in figures $13 \mathrm{~b}$, $13 \mathrm{c}$ and $13 \mathrm{~d}$. It can be concluded that this strategy 
correctly imposes the target minimum length scale on the solid and void phase of the design. The value for the difference ratio $\delta_{\mathrm{OA}}$ for a left-to-right building direction proves that the overhang angle control is effective as well.

In order to demonstrate that the issues with the formation of small support features from section 4.2 are avoided, a bottom-to-top building direction is considered next. This previously led to small features which did not satisfy the minimum length scale, as shown in figure 12a. The resulting optimized design for an imposed bottom-to-top building direction is shown in figure 14a. The compliance is equal to $c=2005$ and the measure of non-discreteness is $M_{\mathrm{nd}}=6.50 \%$. This large increase in compliance is due to the fact that a large amount of material is needed to build a support structure that does not contribute to the stiffness of the design. The difference ratios $\delta_{\mathrm{LV}}=0.00 \%$, $\delta_{\mathrm{LS}}=0.00 \%$ and $\delta_{\mathrm{OA}}=0.00 \%$ of the design are calculated as illustrated in figures $14 \mathrm{~b}, 14 \mathrm{c}$ and $14 \mathrm{~d}$. It can be concluded that the target minimum length scale is satisfied in both the solid and void phase of the design. The zero value for the difference ratio $\delta_{\mathrm{OA}}$ proves that the overhang angle control is effective.

To demonstrate the robustness of the proposed filtering scheme, an extended set of examples has been studied. The optimized designs are shown in figure 15 , where the length scale $R_{\mathrm{S}}$ (used as the filter radius for the open filter) in the solid phase increases from left to right and the length scale $R_{\mathrm{v}}$ (used as the filter radius for the close filter and the additional dilate and erode filters) in the void phase from top to bottom. All tests show near perfect length scale and overhang angle control with values of the difference ratios $\delta_{\mathrm{LV}}, \delta_{\mathrm{LS}}$ and $\delta_{\mathrm{OA}}$ around $0.001 \%$.

The examples considered in this section show that the proposed strategy correctly imposes the target length scale and overhang angle. The values for the difference ratios of the previously described filtering strategies are summarized in table 1 . The difference ratios of the strategy 2 and strategy 3 filtering schemes (described in section 4) represent the examples in which a bottom-to-top building direction is imposed. As previously demonstrated, it is noted that only the proposed filtering scheme is capable of controlling both the length scale and overhang angle simultaneously. Figure 16 shows the convergence history of the optimization problem for the 4 previously described filtering methods. The original minimum compliance problem has a computational cost of $t=0.90 \mathrm{~s}$ per iteration and has

\begin{tabular}{lrrrr}
\hline Filtering scheme & Section & $\delta_{\mathrm{LV}}[\%]$ & $\delta_{\mathrm{LS}}[\%]$ & $\delta_{\mathrm{OA}}[\%]$ \\
\hline \hline original & 2.1 & 1.00 & 2.05 & 0.91 \\
length scale & 3.1 & 0.00 & 0.00 & 0.72 \\
overhang angle & 3.2 & 1.05 & 3.40 & 0.00 \\
combination 1 & 4.1 & 0.27 & 0.57 & 0.00 \\
combination 2 & 4.2 & 0.31 & 0.56 & 9.44 \\
proposed scheme & 4.3 & 0.00 & 0.00 & 0.00 \\
\hline
\end{tabular}

Table 1: Summary of difference ratios for all filtering schemes. Only strategy 3 is capable of controlling both the length scale and overhang angle simultaneously.

the lowest compliance value of $c=1627$. The optimization with an imposed left-to-right building direction has a computational cost of $t=1.03 \mathrm{~s}$ per iteration and a compliance close to the one of the original problem, since the final design shows no significant changes in the topology. The design satisfying the minimum length scale has a higher compliance value due to the added length scale control and a computational cost of $t=0.985 \mathrm{~s}$ per iteration. Adding the length scale and overhang angle control leads to a design with a slightly higher compliance value of $c=1673$, and a computational cost of $t=1.15 \mathrm{~s}$ per iteration. The initial dip in the convergence curves is due to the continuation scheme on the penalization power $p$, which reaches its maximum at around 110 iterations. The relatively small differences in convergence behavior demonstrate that a large set of sequential filters does not lead to any convergence issues or a significant increase in computational cost when using MMA as the optimizer.

Langelaar [25] stated that when applying the overhang angle filter, approximation errors may lead to the formation of gradually decreasing densities for added support features. To avoid these problems, it was proposed to penalize the output of the max operator by increasing the exponent $1 / p$ in equation 15 . This problem does not occur in the case of the proposed filtering scheme. The intermediate densities are removed by the self-penalizing length scale filter, which guides the optimization to a black-and-white solution.

\section{$53 \mathrm{D}$ case study}

\subsection{Problem description}

The practical use of the proposed filtering scheme is demonstrated in this section by considering the design of a single joint of a large tensegrity structure. This problem has previously been studied by the engineering office ARUP, and is documented in [38,39]. Figure 17 shows the original joint, produced in galvanized steel by 


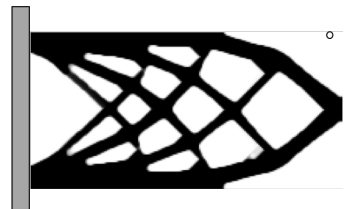

(a) Optimized design; $c=1677, M_{\mathrm{nd}}=5.50 \%$

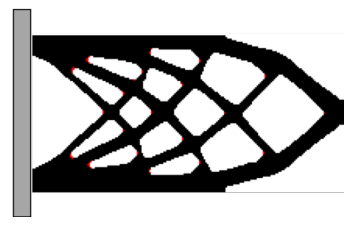

(b) Void length scale check; $\delta_{\mathrm{LV}}=0.27 \%$.

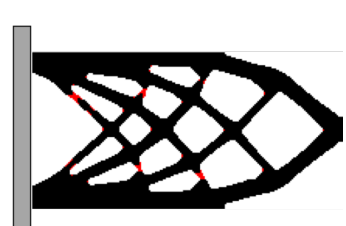

(c) Solid length scale check; $\delta_{\mathrm{LS}}=0.57 \%$.

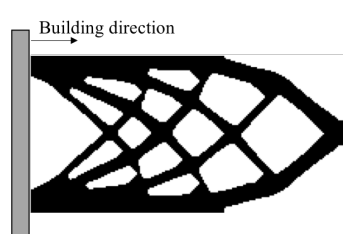

(d) Overhang angle check; $\delta_{\mathrm{OA}}=0.00 \%$.

Fig. 10: Design optimized with a strategy 1 filtering scheme.

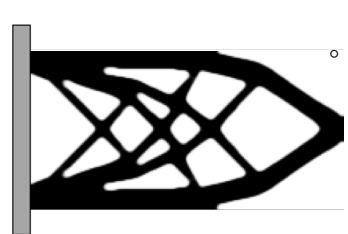

(a) Optimized design; $c=1664, M_{\mathrm{nd}}=6.00 \%$

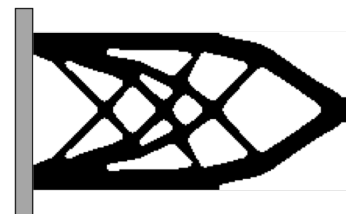

(b) Void length scale check; $\delta_{\mathrm{LV}}=0.00 \%$.

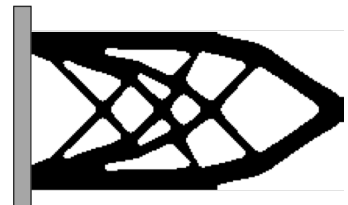

(c) Solid length scale check; $\delta_{\mathrm{LS}}=0.00 \%$.

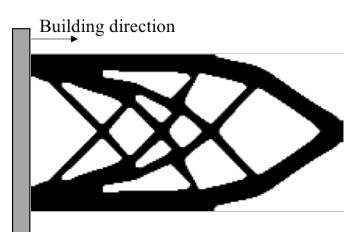

(d) Overhang angle check; $\delta_{\mathrm{OA}}=0.00 \%$.

Fig. 11: Design optimized with the strategy 2 filtering scheme and a left-to-right building direction.

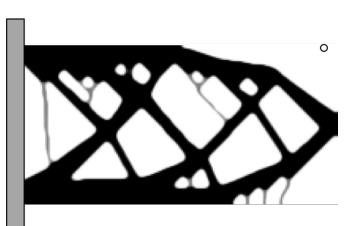

(a) Optimized design; $c=1794, M_{n d}=7.90 \%$

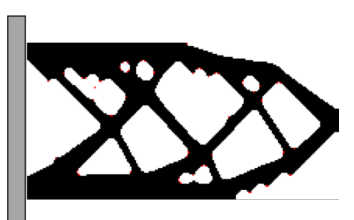

(b) Void length scale check; $\delta_{\mathrm{LV}}=0.31 \%$.

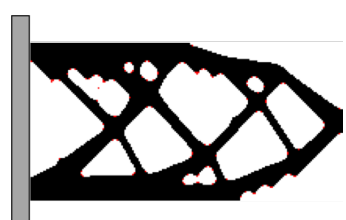

(c) Solid length scale check; $\delta_{\mathrm{LS}}=0.56 \%$.

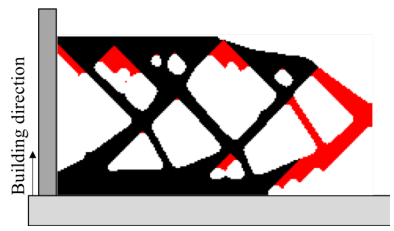

(d) Overhang angle check; $\delta_{\mathrm{OA}}=9.44 \%$.

Fig. 12: Design optimized with the strategy 2 filtering scheme and a bottom-to-top building direction.

welding, compared to the optimized designs, produced in stainless steel by additive manufacturing.

A tensegrity structure is a special type of structure where struts and cables balance each other without the struts ever touching [40]. In this case, the structure consists of around 1200 unique joints where one strut and a set of cables come together. This justifies the use of topology optimization and additive manufacturing to design and produce the joints. The design domain of all 1200 joints is implemented as a parametric design space, presented in figure 18, where the cable orientations can vary. After specifying the design domain, topology optimization is used to design these joints. When all joints are designed, they can be manufactured by metal printing.

Technological constraints of additive manufacturing were not accounted for in the optimized joint from ARUP. A consequence is that all joints need to be postprocessed before manufacturing, which is very time consuming and reduces the effectiveness of additive manufacturing. In this paper, the same joint is optimized taking into account relevant manufacturing constraints, i.e. overhang angle and minimum length scale.

\subsection{Implementation}

The joint is optimized using an adapted version of the $\mathrm{C}++$ framework developed by Aage et al. [41] incorporating the PETSc libraries for parallel computing [42]. This algorithm has been implemented on the thin node section of the High Performance Cluster (HPC) of KU Leuven. This section is equipped with 208 nodes containing two 10-core "Ivy Bridge" Xeon E5-2680v2 CPUs $(2.8 \mathrm{GHz}, 25 \mathrm{MB}$ level 3 cache). The nodes are linked to a QDR Infiniband network, the total memory capacity of this thin node section is $30 \mathrm{~TB}$ and the total peak performance is about 232 Tflops in double precision arithmetic.

The design domain of the tensegrity joint is presented in figure 19. It is a cylinder with a height 


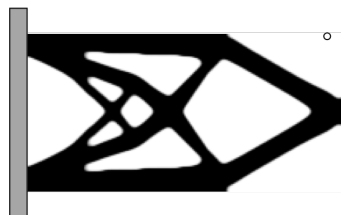

(a) Optimized design; $c=1673, M_{\mathrm{nd}}=5.50 \%$

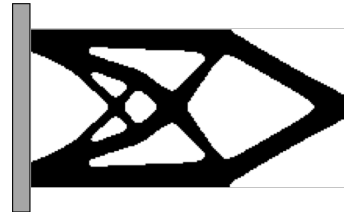

(b) Void length scale check; $\delta_{\mathrm{LV}}=0.00 \%$.

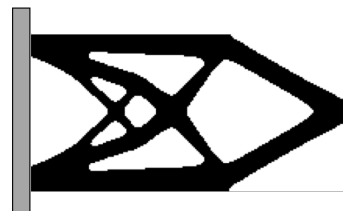

(c) Solid length scale check; $\delta_{\mathrm{LS}}=0.00 \%$.

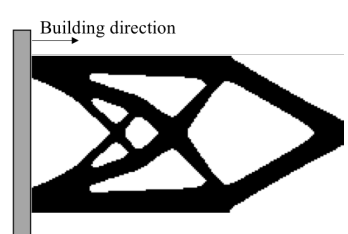

(d) Overhang angle check; $\delta_{\mathrm{OA}}=0.00 \%$.

Fig. 13: Design optimized with strategy 3 filtering scheme and a left-to-right building direction.

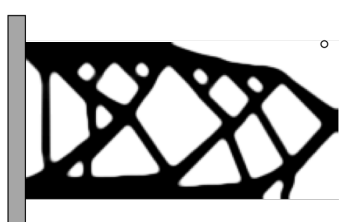

(a) Optimized design; $c=2005, M_{\mathrm{nd}}=6.50 \%$

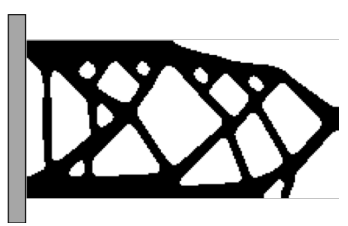

(b) Void length scale check; $\delta_{\mathrm{LV}}=0.00 \%$.

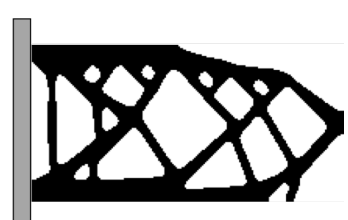

(c) Solid length scale check; $\delta_{\mathrm{LS}}=0.00 \%$.

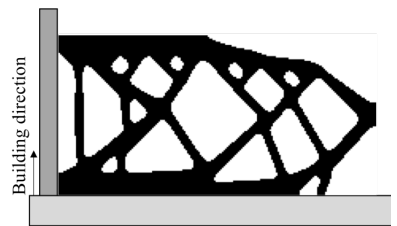

(d) Overhang angle check; $\delta_{\mathrm{OA}}=0.00 \%$.

Fig. 14: Design optimized with strategy 3 filtering scheme and a bottom-to-top building direction.

of $h=255 \mathrm{~mm}$ and a diameter of $d=191.25 \mathrm{~mm}$. The boundary conditions consist of a bottom ring for connection with the strut and six rings to connect the cables. The bottom ring is clamped. It has a height of $h_{\text {ring }}=10 \mathrm{~mm}$ and a radius $r_{\text {ring }}=60 \mathrm{~mm}$. The tension force of the cables is divided over the domain of the six rings with an outer radius $r_{\text {outer }}=25 \mathrm{~mm}$ and an inner radius $r_{\text {inner }}=11 \mathrm{~mm}$. The node is modelled in a Cartesian coordinate system, with the origin at the center of the cylinder's base and the z-axis pointing upwards. The forces of the cables intersect in a single point on the centerline of the cylinder at a height $z=216 \mathrm{~mm}$, so avoiding bending moments in the strut. The directional components of each force vector are summarized in table 2. Around every cable centerline, a cylindrical passive void domain is introduced to save space for tool access. The rings are modeled using passive solid elements.

\begin{tabular}{lrrr}
\hline Ring & Fx $[\mathrm{kN}]$ & Fy $[\mathrm{kN}]$ & Fz $[\mathrm{kN}]$ \\
\hline \hline 1 & -3.47 & 81.50 & 30.35 \\
2 & 5.85 & -44.10 & -24.30 \\
3 & -15.71 & -26.18 & -3.69 \\
4 & -35.10 & -7.56 & -39.96 \\
5 & -63.29 & -17.20 & 19.26 \\
6 & -27.36 & 8.89 & 18.12 \\
\hline
\end{tabular}

Table 2: Summary of directional components of forces acting on each cable ring.
The optimization is performed on a finite element grid of $256 \times 192 \times 192=9437184$ cubic $\mathbb{Q}_{1}$ elements using passive void elements outside the cylindrical design domain. A Young's modulus $E_{0}=180 \mathrm{GPa}$ for the solid phase and $E_{\min }=10^{-9} \mathrm{GPa}$ for the void phase is used, the Poisson's coefficient is set to 0.3 . The compliance of the joint is minimized for a given amount of material. The maximum volume fraction is set to $12 \%$ of the total volume. A target filter radius of $R=3 \mathrm{~mm}$ is used. The modified SIMP interpolation scheme is adopted with penalization power $p=1$ going to $p_{\max }=3$ using a continuation scheme, where $p$ increases with a factor 1.01 in every iteration. A similar continuation scheme is applied to the Heaviside parameter $\beta$, starting at $\beta_{0}=1$ and increasing with a factor 1.01 in every iteration until $\beta \geq 30$. The optimization is performed using the parallel implementation of the Method of Moving Asymptotes (MMA) provided by Aage et al. [43]. Convergence is assumed if the maximum change in element densities between iterations is less than 0.008 .

\subsection{Results}

The results obtained for the 3D optimization problem of the tensegrity joint are presented in this section. First, the joint is optimized using the filtering scheme described in subsection 3.1. Next, overhang angle control is included assuming a bottom-to-top building direction, according to the filtering scheme described in subsection 4.3. The section ends with a brief discussion on the obtained results. The results shown in this sec- 


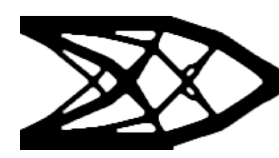
$R_{\mathrm{v}}=0.01 L$ $\delta_{\mathrm{LS}}=0.00 \%$ $\delta_{\mathrm{LV}}=0.00 \%$ $\delta_{\mathrm{OA}}=0.00 \%$

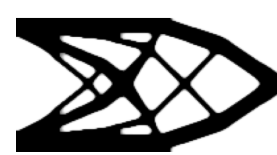

(d) $R_{\mathrm{s}}=0.01 L$ $R_{\mathrm{v}}=0.015 L$

$\delta_{\mathrm{LS}}=0.00 \%$ $\delta_{\mathrm{LV}}=0.00 \%$

$\delta_{\mathrm{OA}}=0.00 \%$

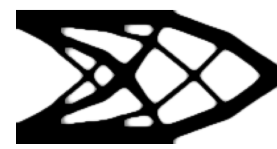

(g) $R_{\mathrm{S}}=0.01 L$ $R_{\mathrm{v}}=0.02 L$

$\delta_{\mathrm{LS}}=0.00 \%$

$\delta_{\mathrm{LV}}=0.00 \%$

$\delta_{\mathrm{OA}}=0.00 \%$ (a) $R_{\mathrm{S}}=0.01 \mathrm{~L}$

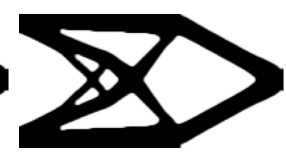

(b) $R_{\mathrm{S}}=0.015 \mathrm{~L}$ $R_{\mathrm{v}}=0.01 L$ $\delta_{\mathrm{LS}}=0.00 \%$ $\delta_{\mathrm{LV}}=0.00 \%$ $\delta_{\mathrm{OA}}=0.00 \%$

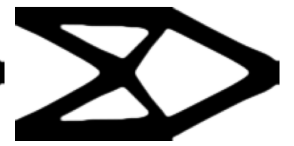

(c) $R_{\mathrm{S}}=0.02 L$ $R_{\mathrm{v}}=0.01 L$ $\delta_{\mathrm{LS}}=0.00 \%$ $\delta_{\mathrm{LV}}=0.00 \%$ $\delta_{\mathrm{OA}}=0.00 \%$

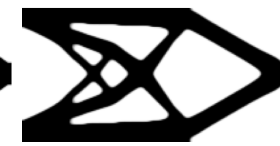

(e) $R_{\mathrm{S}}=0.015 \mathrm{~L}$ $R_{\mathrm{v}}=0.015 L$ $\delta_{\mathrm{LS}}=0.00 \%$ $\delta_{\mathrm{LV}}=0.00 \%$ $\delta_{\mathrm{OA}}=0.00 \%$

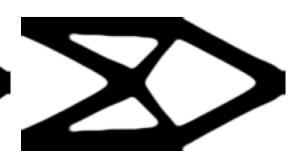

(f) $R_{\mathrm{s}}=0.02 \mathrm{~L}$ $R_{\mathrm{v}}=0.015 L$ $\delta_{\mathrm{LS}}=0.00 \%$ $\delta_{\mathrm{LV}}=0.00 \%$ $\delta_{\mathrm{OA}}=0.00 \%$

Fig. 15: Designs optimized with a strategy 3 filtering scheme and a left-to-right building direction with different values for $R_{\mathrm{s}}$ and $R_{\mathrm{v}}$.

tion are rendered, smoothed versions of the final design. This smoothing operation is performed in Paraview by interpolating the element densities at the nodes.

\section{Without overhang angle control}

The optimized design with length scale control, but without overhang angle control is shown in figure 20 . The compliance is equal to $c=7.87 \times 10^{4} \mathrm{Nm}$ and the measure of non-discreteness is $M_{\mathrm{nd}}=2.50 \%$. The difference ratios $\delta_{\mathrm{LV}}=0.00 \%$ and $\delta_{\mathrm{LS}}=0.00 \%$ demonstrate that the target length scale is correctly imposed on both solid and void phase of the design. These ratios are computed considering the active elements only. The connection elements, bottom ring and cable rings do not satisfy the required length scale, as they are implemented as passive elements and therefore their densities can not be altered by the optimizer. The non-zero value for the difference ratio $\delta_{\mathrm{OA}}=8.50 \%$ proves that the design is not self supporting. Since the overhang angle was not imposed during the optimization process, it is not surprising that such a high value is obtained for $\delta_{\mathrm{OA}}$.

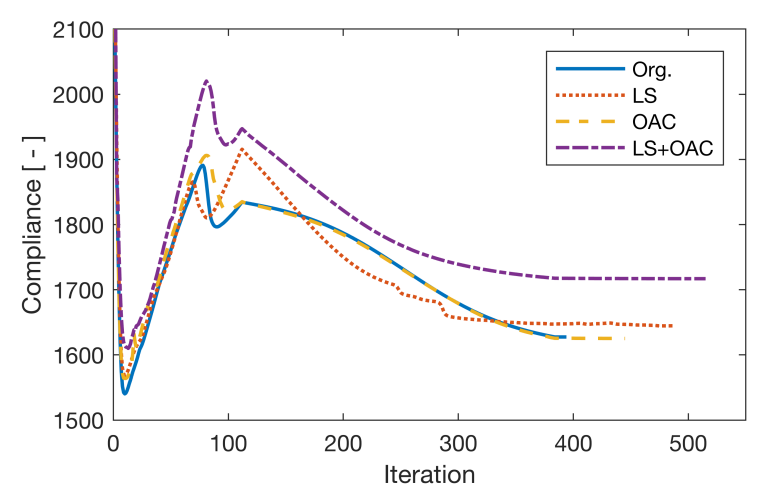

Fig. 16: Convergence plots of the solution of the minimum compliance problem with different filtering strategies; blue: standard minimum compliance problem. $c=1627$ and $t=1.15 \mathrm{~s}$ per iteration; red: imposed minimum length scale. $c=1644$ and $t=1.032 \mathrm{~s}$ per iteration; yellow: imposed leftto-right building direction. $c=1629$ and $t=$ 0.985 s per iteration; purple: proposed filtering scheme. $c=1673$ and $t=1.15$ s per iteration.

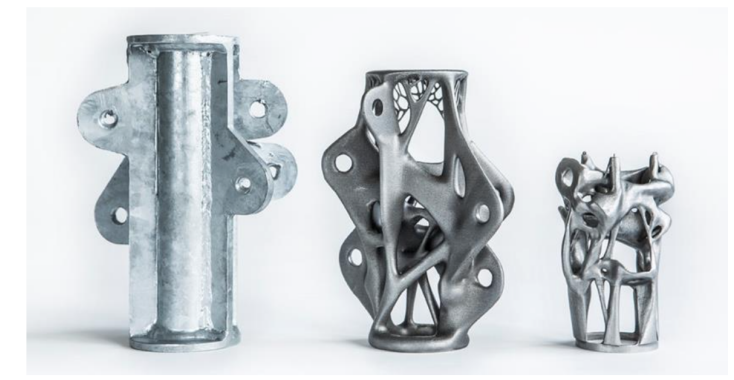

Fig. 17: Resulting topology of the tensegrity joint in three stages of the optimization; (a) traditional joint, (b) optimized joint with standard cable connections, and (c) optimized joint with adapted cable connections; source: [39].

\section{With overhang angle control}

The optimized design with length scale control and a bottom-to-top building direction is shown in figure 21 . The compliance is equal to $c=10.7 \times 10^{4} \mathrm{Nm}$, which is slightly higher than the previous case due to the additional overhang angle control. The measure of nondiscreteness is equal to $M_{\mathrm{nd}}=4.50 \%$. The difference ratios $\delta_{\mathrm{LS}}=0.00 \%, \delta_{\mathrm{LV}}=0.00 \%$ and $\delta_{\mathrm{OA}}=0.00 \%$ suggest a correctly imposed length scale and overhang angle. 


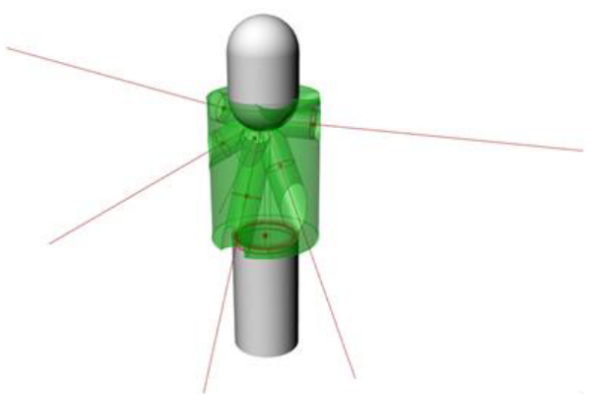

Fig. 18: Parametric design space of the joint to allow for different configurations; source: [39].

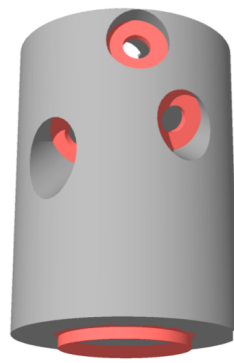

(a)

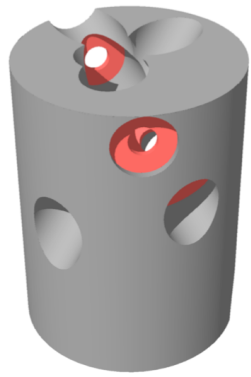

(b)
Fig. 19: Domain and boundary conditions of the tensegrity joint; (a) bottom view; (b) topview.
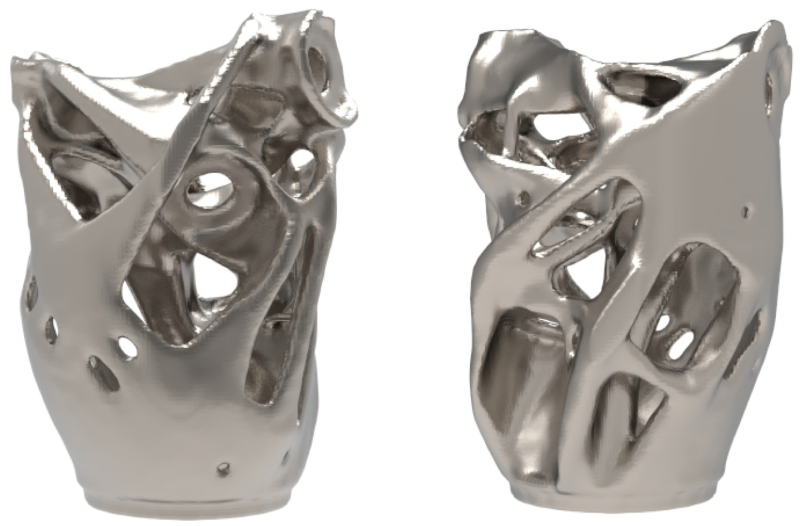

Fig. 20: Different views of the optimized tensegrity joint design without overhang angle control control with: $R_{\text {solid }}=3 \mathrm{~mm}, R_{\text {void }}=3 \mathrm{~mm}, c=7.87 \times 10^{4} \mathrm{Nm}$, $M_{\mathrm{nd}}=2.50 \%, \delta_{\mathrm{LS}}=0.00 \%, \delta_{\mathrm{LV}}=0.00 \%, \delta_{\mathrm{OA}}=$ $8.50 \%$.

\subsection{Discussion}

The optimized designs show that the proposed strategy successfully imposes a target length scale and overhang angle, even for complex 3D designs. The convergence plots of both optimization processes, presented in figure
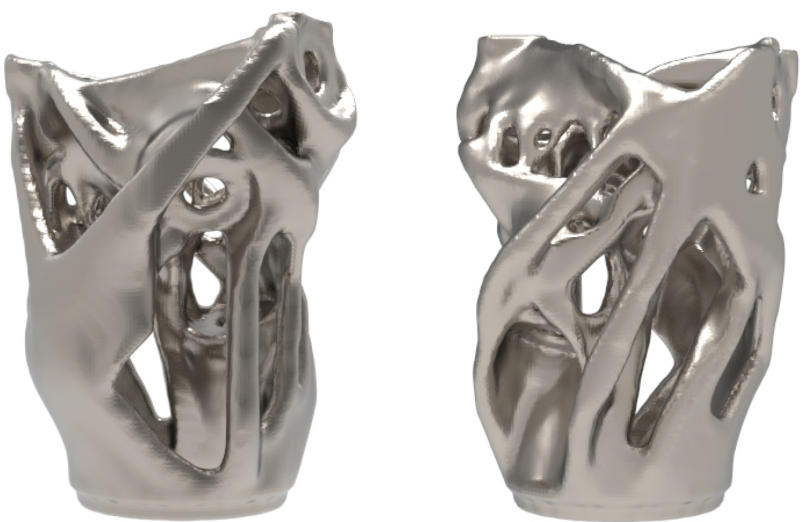

Fig. 21: Different views of the optimized tensegrity joint design with a bottom-to-top building direction with: $R_{\text {solid }}=3 \mathrm{~mm}, R_{\text {void }}=3 \mathrm{~mm}, c=10.7 \times 10^{4}, M_{\mathrm{nd}}=$ $4.50 \%, \delta_{\mathrm{LS}}=0.00 \%, \delta_{\mathrm{LV}}=0.00 \%, \delta_{\mathrm{OA}}=0.00 \%$.

22 , demonstrate that the added filtering scheme does not have a significant impact on the convergence speed and stability of the optimization process. The initial increase of the compliance in the curves is due to the continuation scheme on the penalization power $p$, this value reaches its maximum of $p=3$ at around 110 iterations. After the peak, the convergence plot shows some instabilities in the optimization with overhang angle control. This can be explained by the continuously increasing Heaviside parameter; when the design is evolving to a black-and-white solution, some features that previously satisfied the minimum length scale will become too small. Removal of these features by the length scale control filters results in a compliance jump.

It is noted that the imposed length scale and overhang angle are not satisfied in the bottom ring and cable rings, as they are implemented as passive elements and can not be changed during optimization. As a consequence, the temporary support structure can not be completely eliminated in this case, but it can be reduced to a minimum.

To validate the proposed filtering scheme, the $3 \mathrm{D}$ examples have been checked using an open source printing software package and a professional software package. If only geometric limitations of the $3 \mathrm{D}$ printer are considered, the support structure is indeed eliminated (except underneath the passive elements). This is demonstrated in figure 23 using a Fused Deposition Modeling test print in PLA. However, when assuming all limitations of a 3D metal printer, the amount of support structure is not reduced as much. This is due to thermal stresses that are induced during the printing process. 


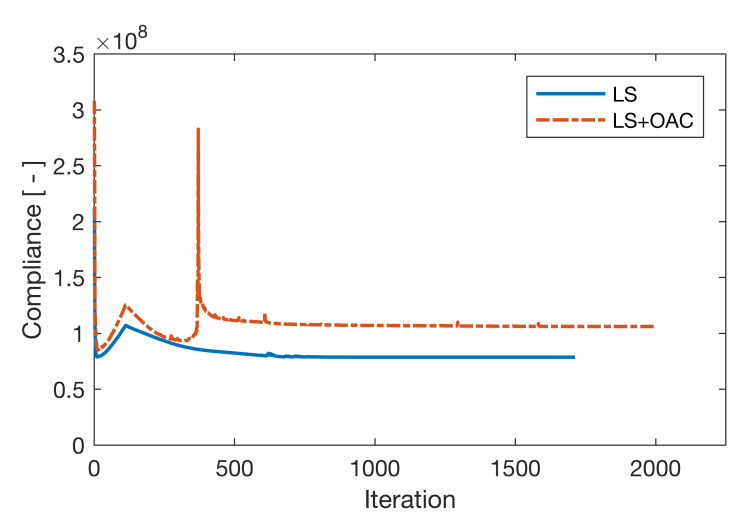

Fig. 22: Convergence plot of the tensegrity joint; the blue curve represents the problem without overhang angle control with a final compliance of $c=7,87 \times 10^{4}$ $\mathrm{Nm}$; the red curve represents the problem with an imposed building direction with a final compliance of $c=10,7 \times 10^{4} \mathrm{Nm}$.

These stresses can result in deformations which can destroy the printed element if they are not inhibited by a sufficient amount of support structure. This means that only optimizing for geometric constraints, such as overhang angles, is just a first step to reduce the amount of support structure.
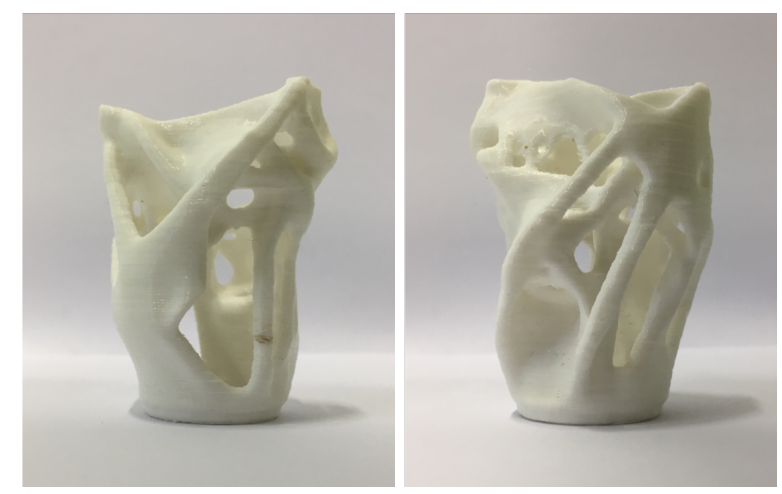

Fig. 23: Different views of the printed tensegrity joint with a bottom-to-top building direction; no additional support structure has been used.

\section{Conclusion}

This paper addresses minimum length scale and maximum overhang angle control in minimum compliance topology optimization for additive manufacturing. Simply combining existing solutions for both individual problems is not sufficient to control both length scale and overhang angle simultaneously. Two straightforward combinations are tested in this paper to demonstrate this. The first is a minimum length scale filter followed by a maximum overhang angle filter; the second is a maximum overhang angle filter followed by a minimum length scale filter. In both cases, the second filter neutralizes the effect of the first, leading to a violation of one of the requirements. In order to correctly obtain the desired control, this paper presented a new, more complex filtering scheme for minimum compliance problems. The proposed filtering strategy is based on a maximum overhang angle filter followed by a minimum length scale filter, where additional erode and dilate filters are applied before and after the former in order to avoid that its effect is neutralized by the latter. In addition, imposing a minimum length scale in the void phase of the design proved to provide the added advantage of a controllable maximum fillet radius of the design's interior corners. Validation on a 2D benchmark problem and a complex real life 3D design problem demonstrated that the filtering strategy successfully provides length scale and overhang angle control to the optimization process, even if more complex geometries and non-trivial build directions are considered.

Acknowledgements The first author is a doctoral fellow of the Research Foundation Flanders (FWO). The financial support is gratefully acknowledged. The authors also acknowledge Shibo Ren from ARUP for sharing the information required to perform the $3 \mathrm{D}$ case study.

\section{References}

1. D. Rosen. Design for additive manufacturing: Past, present, and future directions. Journal of Mechanical Design, 136, September 2014.

2. M.P. Bendsøe. Optimal shape design as a material distribution problem. Structural and Multidisciplinary $O p$ timization, 1(4):193-202, 1989.

3. M. Zhou and G.I.N. Rozvany. The COC algorithm, part II: Topological, geometrical and generalized shape optimization. Computer Methods in Applied Mechanics and Engineering, 89(1-3):309-336, 1991.

4. A.R. Díaz and O. Sigmund. Checkerboard patterns in layout optimization. Structural Optimization, 10(1):40$45,1995$.

5. C.S. Jog and R.B. Haber. Stability of finite element models for distributed-parameter optimization and topology design. Computer Methods in Applied Mechanics and Engineering, 130(3-4):203-226, 1996.

6. T.E. Bruns and D.A. Tortorelli. Topology optimization of non-linear elastic structures and compliant mechanisms. Computer Methods in Applied Mechanics and Engineering, 190(26-27):3443-3459, 2001.

7. B. Bourdin. Filters in topology optimization. International Journal for Numerical Methods in Engineering, 50(9):2143-2158, 2001. 
8. J.K. Guest, J.H. Prevost, and T. Belytschko. Achieving minimum length scale in topology optimization using nodal design variables and projection functions. International Journal for Numerical Methods in Engineering, 61(2):238-254, 2004.

9. S.L. Xu, Y.W. Cai, and G.D. Cheng. Volume preserving nonlinear density filter based on Heaviside functions. Structural and Multidisciplinary Optimization, 41(4):495-505, 2010.

10. O. Sigmund. Morphology-based black and white filters for topology optimization. Structural and Multidisciplinary Optimization, 33(4-5):401-424, 2007.

11. W.K. Pratt. Digital image processing. Wiley, 1991.

12. F. Wang, B.S. Lazarov, and O. Sigmund. On projection methods, convergence and robust formulations in topology optimization. Structural and Multidisciplinary Optimization, 43(6):767-784, 2011.

13. B.S. Lazarov, F. Wang, and O. Sigmund. Length scale and manufacturability in density-based topology optimization. Archive of Applied Mechanics, 86(1-2):189$218,2016$.

14. L. Hägg and E. Wadbro. Nonlinear filters in topology optimization: existence of solutions and efficient implementation for minimum compliance problems. Structural and Multidisciplinary Optimization, (55):1017-1028, 2017.

15. G. Allaire, F. Jouve, and G. Michailidis. Thickness control in structural optimization via a level set method. Structural and Multidisciplinary Optimization, (53):1349-1382, 2016.

16. W. Zhang, D. Li, J. Zhang, and X. Guo. Minimum length scale control in structural topology optimization based on the moving morphable components (mmc) approach. Computer Methods in Applied Mechanics and Engineering, (311):327-355, 2016.

17. I. Gibson, D. Rosen, and B. Stucker. Additive Manufacturing Technologies. Springer, 2015.

18. E. Atzeni and A. Salmi. Economics of additive manufacturing for end-usable metal parts. Int. J. Adv. Manuf. Technol., 62:1147-1155, 2012.

19. R. Becker, A Grzesiak, and A Henning. Rethink assembly design. Assembly Automation, 25(4):262-266, 2005.

20. D. Cormier and T Harrysson, O. Mahale. Rapid manufacturing in the 21st century. Journal of the Chinese Institute of Industrial Engineers, 20(3):193-202, 2003.

21. J. Liu and Y. Ma. A survey of manufacturing oriented topology optimization methods. Advances in Engineering Software, pages 161-175, 2016.

22. D. Brackett, I. Ashcroft, and R. Hague. Topology optimization for additive manufacturing. In Proceedings of the Solid Freeform Fabrication Symposium, Austin, TX, US, pages 348-362, 2011.

23. A.T. Gaynor and J.K. Guest. Topology optimization for additive manufacturing: considering maximum overhang constraint. In 15th AIAA/ISSMO Multidisciplinary Analysis and Optimization Conference, pages 1620,2014

24. X. Qian. Undercut and overhang angle control in topology optimization: A density gradient based integral approach. International Journal for Numerical Methods in Engineering, 2017.

25. M. Langelaar. An additive manufacturing filter for topology optimization of print-ready designs. Structural and Multidisciplinary Optimization, July 2016.

26. D Wang, Y Yang, Z Yi, and X Su. Research on the fabricating quality optimization of the overhanging surface in slm process. Int J Adv Manuf Technol, 65:1471-1484, 2013.
27. J Kranz, D Herzog, and C Emmelmann. Design guidelines for laser additive manufacturing of lightweight structures in tial6v4. Journal of Laser Applications, 27, 2015.

28. A. Mirzendehdel and K. Suresh. Support structure constrained topology optimization for additive manufacturing. Computer-Aided Design, pages 1-13, 2016.

29. M. Langelaar. Topology optimization of 3D selfsupporting structures for additive manufacturing. $A d$ ditive Manufacturing, 12(A):60-70, 2016.

30. G. Allaire, C. Dapogny, A. Faure, and G. Michailidis. Shape optimization of a layer by layer mechanical constraint for additive manufacturing. C. R. Acad. Sci. Paris, Ser. I, pages 699-717, 2017.

31. G. Allaire, C. Dapogny, A. Estevez, A. Faure, and G. Michailidis. Structural optimization under overhang constraints imposed by additive manufacturing technologies. Journal of Computational Physics, pages 295-328, 2017.

32. O. Amir and Y. Mass. Topology optimization for staged construction. Structural and Multidisciplinary Optimization, pages 1679-1694, 2018.

33. M.P. Bendsøe and O. Sigmund. Topology optimization: theory, methods and applications. Springer, Berlin, second edition, 2004.

34. O. Sigmund and J. Petersson. Numerical instabilities in topology optimization: A survey on procedures dealing with checkerboards, mesh-dependencies and local minima. Structural Optimization, 16(1):68-75, 1998.

35. K. Svanberg. The method of moving asymptotes - a new method for structural optimization. International Journal for Numerical Methods in Engineering, 24(2):359373, 1987.

36. O. Amir and B. Lazarov. Achieving stress-constrained topological designs via length scale control. In review.

37. M. Schevenels and O. Sigmund. On the implementation and effectiveness of morphological close-open and openclose filters for topology optimization. Structural and Multidisciplinary Optimization, 54(1):15-21, 2016.

38. S. Galjaard, S. Hofman, and S. Ren. New opportunities to optimize structural designs in metal by using additive manufacturing. In Advances in Architectural Geometry, pages 79-93. Springer, 2014.

39. S. Galjaard, S. Hofman, N. Perry, and S. Ren. Optimizing structural building elements in metal by using additive manufacturing. In Proceedings of the International Association for Shell and Spatial Structures (IASS), 2015.

40. K. Snelson. The art of tensegrity. International Journal of Space Structures, 27(2 and 3), 2012.

41. N. Aage and B.S. Lazarov. Parallel framework for topology optimization using the method of moving asymptotes. Structural and Multidisciplinary Optimization, 47:493-505, 2013

42. S. Balay, S. Abhyankar, M. Adams, J. Brown, P. Brune, K. Buschelman, L. Dalcin, V. Eijkhout, W. Gropp, D. Kaushik, and M. Knepley. Petsc users manual. Technical Report ANL-95/11 - Revision 3.7, Argonne National Laboratory, 2016.

43. N. Aage, E. Andreassen, and B.S. Lazarov. Topology optimization using PETSc: An easy-to-use, fully parallel, open source topology optimization framework. Structural and Multidisciplinary Optimization, 51(3):565-572, 2014.

\section{A Sensitivity analysis}

The sensitivity analysis of the different filtering schemes considered in this paper are elaborated in the following subsec- 
tions, where the derivative of a vector $\mathbf{y}$ with respect to a vector $\mathbf{x}$ is denoted as $\frac{\partial \mathbf{y}}{\partial \mathbf{x}}$, which represents a matrix where the $(i, j)^{t h}$ elements equals:

$$
\left[\frac{\partial \mathbf{y}}{\partial \mathbf{x}}\right]_{i j}=\frac{\partial y_{i}}{\partial x_{j}}
$$

\section{A.1 Minimum compliance topology optimization}

The relation between the design variables $\rho$ and the compliance $c$ is given by the filtering scheme in equation (7), the sensitivity $\frac{\partial c}{\partial \rho}$ of the objective function $c(\boldsymbol{\rho})$ with respect design variables $\rho$ for minimum compliance topology optimization is computed by applying the chain rule 2 times:

$$
\frac{\partial c}{\partial \boldsymbol{\rho}}=\frac{\partial c}{\partial \overline{\boldsymbol{\rho}}} \frac{\partial \overline{\boldsymbol{\rho}}}{\partial \tilde{\boldsymbol{\rho}}} \frac{\partial \tilde{\boldsymbol{\rho}}}{\partial \boldsymbol{\rho}}
$$

The sensitivity $\frac{\partial c}{\partial \overline{\boldsymbol{\rho}}}$ is computed using the adjoint variable method:

$\frac{\partial c}{\partial \bar{\rho}_{e}}=-\boldsymbol{\lambda}^{T} \frac{\partial \mathbf{K}}{\partial \bar{\rho}_{e}} \mathbf{u}$

where the adjoint variable $\boldsymbol{\lambda}$ is obtained by by solving $\mathbf{K} \boldsymbol{\lambda}=\mathbf{f}$, this results in $\boldsymbol{\lambda}=\mathbf{u}$ for the minimum compliance problem.

The sensitivity $\frac{\partial \bar{\rho}}{\partial \tilde{\rho}}$ of the Heaviside projection, equation (6), is given by:

$$
\frac{\partial \overline{\boldsymbol{\rho}}}{\partial \tilde{\boldsymbol{\rho}}}=\operatorname{diag}\left(\frac{\beta(\operatorname{sech}(\beta(\tilde{\boldsymbol{\rho}}-\eta)))}{\tanh (\beta \eta)+\tanh (\beta(1-\eta))}\right)
$$

The density filter in equation (4) is a linear operator that can be expressed as:

$\tilde{\boldsymbol{\rho}}=\mathbf{H}^{R} \boldsymbol{\rho}$

where the coefficient matrix $\mathbf{H}^{R}$ consists of elements $H_{i j}^{R}=$ $\frac{h_{i j}^{R}}{\sum_{j \in \mathbb{N}_{e}} h_{i k}^{R}}$, resulting in a sensitivity $\frac{\partial \tilde{\rho}}{\partial \boldsymbol{\rho}}$ of:

$\frac{\partial \tilde{\rho}}{\partial \boldsymbol{\rho}}=\mathbf{H}^{R}$

The sensitivity $\frac{\partial V}{\partial \rho}$ of the constraint function with respect to the design variables $\rho$ is obtained in a similar way as the sensitivities of the complaince.

\section{A.2 Length scale control}

Following the filtering scheme presented in equation (11), the sensitivity $\frac{\partial c}{\partial \boldsymbol{\rho}}$ of the objective function $c(\boldsymbol{\rho})$ with respect to the design variables $\rho$ for minimum compliance topology optimization with length scale control is computed by applying the chain rule 2 times:

$$
\frac{\partial c}{\partial \boldsymbol{\rho}}=\frac{\partial c}{\partial \hat{\hat{\rho}}} \frac{\partial \hat{\tilde{\rho}}}{\partial \check{\boldsymbol{\rho}}} \frac{\partial \check{\boldsymbol{\rho}}}{\partial \boldsymbol{\rho}}
$$

where the sensitivity $\frac{\partial c}{\partial \tilde{\tilde{\rho}}}$ of the objective function with respect to the design variables $\hat{\tilde{\rho}}$ is computed using the adjoint variable method described in equation 25 , and

$$
\begin{aligned}
& \frac{\partial \hat{\tilde{\boldsymbol{\rho}}}}{\partial \check{\boldsymbol{\rho}}}=\operatorname{diag}\left(\frac{\beta\left(\operatorname{sech}\left(\beta\left(\check{\boldsymbol{\rho}}-\eta_{\mathrm{d}}\right)\right)\right)}{\tanh \left(\beta \eta_{\mathrm{d}}\right)+\tanh \left(\beta\left(1-\eta_{\mathrm{d}}\right)\right)}\right) \mathbf{H}^{R}, \\
& \frac{\partial \check{\boldsymbol{\rho}}}{\partial \boldsymbol{\rho}}=\operatorname{diag}\left(\frac{\beta\left(\operatorname{sech}\left(\beta\left(\boldsymbol{\rho}-\eta_{\mathrm{e}}\right)\right)\right)}{\tanh \left(\beta \eta_{\mathrm{e}}\right)+\tanh \left(\beta\left(1-\eta_{\mathrm{e}}\right)\right)}\right) \mathbf{H}^{R}
\end{aligned}
$$

\section{A.3 Overhang angle control}

Following the filtering scheme presented in equation (16), the sensitivity $\frac{\partial c}{\partial \rho}$ of the objective function $c(\boldsymbol{\rho})$ with respect to the design variables $\rho$ for minimum compliance topology optimization with overhang angle control can be obtained via direct differentiation by applying the chain rule 2 times:

$\frac{\partial c}{\partial \boldsymbol{\rho}}=\frac{\partial c}{\partial \hat{\bar{\rho}}} \frac{\partial \hat{\bar{\rho}}}{\partial \overline{\boldsymbol{\rho}}} \frac{\partial \overline{\boldsymbol{\rho}}}{\partial \boldsymbol{\rho}}$

where $\frac{\partial \bar{\rho}}{\partial \rho}$ is calculated along the same lines as equation (30) and (31) with a threshold $\eta=0.5$. However, $\frac{\partial \hat{\rho}}{\partial \bar{\rho}}$ is a densely populated matrix, as the design variables $\hat{\bar{\rho}}$ in a specific layer depend on the blueprint densities $\bar{\rho}$ of all underlying elements. Alternatively, the sensitivity $\frac{\partial c}{\partial \rho}$ is obtained as:

$\frac{\partial c}{\partial \boldsymbol{\rho}}=\frac{\partial c}{\partial \bar{\rho}} \frac{\partial \bar{\rho}}{\partial \boldsymbol{\rho}}$

where $\frac{\partial c}{\partial \bar{\rho}}$ is efficiently obtained using an adjoint formulation proposed by Langelaar [25]. Collecting the blueprint densities of all elements of layer $k$ in a vector $\overline{\boldsymbol{\rho}}_{k}$ and the printed densities in a vector $\hat{\boldsymbol{\rho}}_{k}$, the latter can be expressed as:

$\hat{\overline{\boldsymbol{\rho}}}_{k}=\breve{\mathbf{s}}_{k}\left(\overline{\boldsymbol{\rho}}_{k}, \hat{\overline{\boldsymbol{\rho}}}_{k-1}\right)$,

where, for $k>1$, the definition of the operator $\breve{\mathbf{s}}_{k}$ immediately follows from equation (14) and (15):

$\breve{\mathbf{s}}_{k}=\frac{1}{2}\left(\overline{\boldsymbol{\rho}}_{k}+\hat{\overline{\boldsymbol{\rho}}}_{k}^{S}-\left(\left(\overline{\boldsymbol{\rho}}_{k}-\hat{\overline{\boldsymbol{\rho}}}_{k}^{S}\right)^{2}+\epsilon\right)^{\frac{1}{2}}+\sqrt{\epsilon}\right)$

For $k=1, \breve{\mathbf{s}}_{k}$ simply returns $\overline{\boldsymbol{\rho}}_{k}$, so that $\frac{\partial \breve{\mathbf{s}}_{1}}{\partial \overline{\boldsymbol{\rho}}_{1}}=\mathbf{I}$ and $\frac{\partial \breve{\mathbf{s}}_{1}}{\partial \hat{\boldsymbol{\rho}}_{0}}=\mathbf{0}$.

The compliance can now be expressed as:

$\tilde{c}=c(\hat{\boldsymbol{\rho}}(\overline{\boldsymbol{\rho}}))+\sum_{k=1}^{n_{k}} \boldsymbol{\lambda}_{k}^{T}\left(\breve{\mathbf{s}}_{k}\left(\overline{\boldsymbol{\rho}}_{k}, \hat{\boldsymbol{\rho}}_{k-1}\right)-\hat{\boldsymbol{\rho}}_{k}\right)$,

where $\boldsymbol{\lambda}_{k}$ is a vector with Lagrange multipliers. Differentiation of (36) with respect to the design variables $\bar{\rho}_{l}$ results in:

$$
\begin{aligned}
\frac{\partial \tilde{c}}{\partial \overline{\boldsymbol{\rho}}_{l}}= & \sum_{k=1}^{n_{k}}\left(\frac{\partial c}{\partial \hat{\overline{\boldsymbol{\rho}}}_{k}} \frac{\partial \hat{\overline{\boldsymbol{\rho}}}_{k}}{\partial \overline{\boldsymbol{\rho}}_{l}}+\ldots\right. \\
& \left.\boldsymbol{\lambda}_{k}^{T}\left(\frac{\partial \breve{\mathbf{s}}_{k}}{\partial \overline{\boldsymbol{\rho}}_{l}}+\frac{\partial \breve{\mathbf{s}}_{k}}{\partial \hat{\overline{\boldsymbol{\rho}}}_{k-1}} \frac{\partial \hat{\overline{\boldsymbol{\rho}}}_{k-1}}{\partial \overline{\boldsymbol{\rho}}_{l}}-\frac{\partial \hat{\overline{\boldsymbol{\rho}}}_{k}}{\partial \overline{\boldsymbol{\rho}}_{l}}\right)\right),
\end{aligned}
$$

where $n_{k}$ is the number of layers. As printed densities only depend on blueprint densities in underlying layers, $\frac{\partial \hat{\boldsymbol{\rho}}_{k}}{\partial \overline{\boldsymbol{\rho}}_{l}}=0$ 
for $k<l$, thus, terms in the summations with $k<l$ will disappear. Taking terms with $k=l$ outside of the summations, and using $\frac{\partial \hat{\boldsymbol{\rho}}_{l}}{\partial \overline{\boldsymbol{\rho}}_{l}}=\frac{\partial \breve{\mathbf{s}}_{l}}{\partial \overline{\boldsymbol{\rho}}_{l}}$, gives:

$$
\begin{aligned}
\frac{\partial \tilde{c}}{\partial \overline{\boldsymbol{\rho}}_{l}}= & \frac{\partial c}{\partial \hat{\overline{\boldsymbol{\rho}}}_{l}} \frac{\partial \hat{\overline{\boldsymbol{\rho}}}_{l}}{\partial \overline{\boldsymbol{\rho}}_{l}}+\ldots \\
& \sum_{k=l+1}^{n_{k}}\left(\left(\frac{\partial c}{\partial \hat{\bar{\rho}}_{k}}-\boldsymbol{\lambda}_{k}^{T}\right) \frac{\partial \hat{\boldsymbol{\rho}}_{k}}{\partial \overline{\boldsymbol{\rho}}_{l}}+\boldsymbol{\lambda}_{k}^{T} \frac{\partial \breve{\mathbf{s}}_{k}}{\partial \hat{\overline{\boldsymbol{\rho}}}_{k-1}} \frac{\partial \hat{\overline{\boldsymbol{\rho}}}_{k-1}}{\partial \overline{\boldsymbol{\rho}}_{l}}\right) .
\end{aligned}
$$

Next, the last term in the summation is written as a separate sum and the first term $(k=l+1)$ is taken out:

$$
\begin{aligned}
& \frac{\partial \tilde{c}}{\partial \overline{\boldsymbol{\rho}}_{l}}= \frac{\partial c}{\partial \hat{\bar{\rho}}_{l}} \frac{\partial \hat{\boldsymbol{\rho}}_{l}}{\partial \overline{\boldsymbol{\rho}}_{l}}+\sum_{k=l+1}^{n_{k}}\left(\left(\frac{\partial c}{\partial \hat{\bar{\rho}}_{k}}-\boldsymbol{\lambda}_{k}^{T}\right) \frac{\partial \hat{\boldsymbol{\rho}}_{k}}{\partial \overline{\boldsymbol{\rho}}_{l}}\right)+\ldots \\
& \boldsymbol{\lambda}_{l+1}^{T} \frac{\partial \breve{\mathbf{s}}_{l+1}}{\partial \hat{\bar{\rho}}_{l}} \frac{\partial \hat{\overline{\boldsymbol{\rho}}}_{l}}{\partial \overline{\boldsymbol{\rho}}_{l}}+\sum_{k=l+2}^{n_{k}} \boldsymbol{\lambda}_{k}^{T} \frac{\partial \breve{\mathbf{s}}_{k}}{\partial \hat{\overline{\boldsymbol{\rho}}}_{k-1}} \frac{\partial \hat{\overline{\boldsymbol{\rho}}}_{k-1}}{\partial \overline{\boldsymbol{\rho}}_{l}} .
\end{aligned}
$$

By reindexing, the last sum can be changed into a summation from $k=l+1 \rightarrow n_{k}-1$. Both sums get the same limits by taking the last term $k=n_{k}$ out of the summation. Using $\frac{\partial \hat{\boldsymbol{\rho}}_{l}}{\partial \overline{\boldsymbol{\rho}}_{l}}=\frac{\partial \breve{\mathbf{s}}_{l}}{\partial \overline{\boldsymbol{\rho}}_{l}}$ and recombining summations gives:

$$
\begin{aligned}
\frac{\partial \tilde{c}}{\partial \overline{\boldsymbol{\rho}}_{l}}= & \left(\frac{\partial c}{\partial \hat{\boldsymbol{\rho}}_{l}}+\boldsymbol{\lambda}_{l+1}^{T} \frac{\partial \breve{\mathbf{s}}_{l+1}}{\partial \hat{\boldsymbol{\rho}}_{l}}\right) \frac{\partial \breve{\mathbf{s}}_{l}}{\partial \overline{\boldsymbol{\rho}}_{l}}+\left(\frac{\partial c}{\partial \hat{\boldsymbol{\rho}}_{n_{k}}}-\boldsymbol{\lambda}_{n_{k}}^{T}\right) \frac{\partial \hat{\overline{\boldsymbol{\rho}}}_{n_{k}}}{\partial \overline{\boldsymbol{\rho}}_{l}}+\ldots \\
& \sum_{k=l+1}^{n_{k}-1}\left(\frac{\partial c}{\partial \hat{\overline{\boldsymbol{\rho}}}_{k}}-\boldsymbol{\lambda}_{k}^{T}+\boldsymbol{\lambda}_{k+1}^{T} \frac{\partial \breve{\mathbf{s}}_{k+1}}{\partial \hat{\boldsymbol{\rho}}_{k}}\right) \frac{\partial \hat{\boldsymbol{\rho}}_{k}}{\partial \overline{\boldsymbol{\rho}}_{l}}
\end{aligned}
$$

The computation of the densely populated matrix $\frac{\partial \hat{\boldsymbol{\rho}}_{k}}{\partial \overline{\boldsymbol{\rho}}_{l}}$ can be avoided if the Langrange multipliers are choses as:

$$
\begin{aligned}
\boldsymbol{\lambda}_{l}^{T} & =\frac{\partial c}{\partial \hat{\overline{\boldsymbol{\rho}}}_{l}}+\boldsymbol{\lambda}_{l+1}^{T} \frac{\partial \breve{\mathbf{s}}_{l+1}}{\partial \hat{\bar{\rho}}_{l}} \quad \text { for } 1 \leq l \leq n_{k}, \\
\boldsymbol{\lambda}_{n_{k}}^{T} & =\frac{\partial c}{\partial \hat{\overline{\boldsymbol{\rho}}}_{n_{k}}}
\end{aligned}
$$

Each multiplier depends on the one associated with the layer above. This means that the evaluation starts at the top laver and proceeds downwards. With Lagrange multipliers defined by (41), the sensitivities of the response $c$ follow from (40) as:

$$
\frac{\partial c}{\partial \overline{\boldsymbol{\rho}}_{l}}=\frac{\partial \tilde{c}}{\partial \overline{\boldsymbol{\rho}}_{l}}=\left(\frac{\partial c}{\partial \hat{\overline{\boldsymbol{\rho}}}_{l}}+\boldsymbol{\lambda}_{l+1}^{T} \frac{\partial \breve{\mathbf{s}}_{l+1}}{\partial \hat{\overline{\boldsymbol{\rho}}}_{l}}\right) \frac{\partial \breve{\mathbf{s}}_{l}}{\partial \overline{\boldsymbol{\rho}}_{l}}=\boldsymbol{\lambda}_{l}^{T} \frac{\partial \breve{\mathbf{s}}_{l}}{\partial \overline{\boldsymbol{\rho}}_{l}}
$$

The sensitivity $\frac{\partial c}{\partial \hat{\rho}}$ of the objective function $c$ with respect to the printed densities $\hat{\boldsymbol{\rho}}$ is calculated using the adjoint approach described by equation (25), and the sensitivity $\frac{\partial \breve{s}}{\partial \bar{\rho}}$ follows immediately from the differentiation of equations (14) and (15).

Finally, the sensitivity $\frac{\partial c}{\partial \bar{\rho}}$ needed in equation (33) is obtained by concatenating the derivatives $\frac{\partial c}{\partial \overline{\boldsymbol{\rho}}_{l}}$ for all individual layers.

A.4 Combined length scale and overhang angle control

Following the filtering scheme presented in equation (22), the sensitivity $\frac{\partial c}{\partial \rho}$ of the objective function $c$ with respect to the design variables $\boldsymbol{\rho}$ for minimum compliance topology optimization with length scale and overhang angle control (strategy 3) can be obtained via direct differentiation by applying the chain rule 6 times:

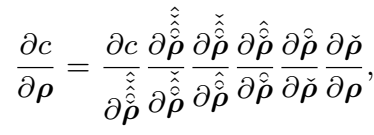

where $\frac{\partial \check{\rho}}{\partial \rho}$ is calculated following the same principle as equation (31). In this case, the term $\frac{\partial \stackrel{\circ}{\partial}}{\partial \check{\rho}}$ is a densely populated matrix, as the design variables $\tilde{\rho}$ in a specific layer depend on the blueprint densities $\check{\rho}$ of all underlying elements. In order to avoid the calculation of this densely populated matrix, the sensitivity $\frac{\partial c}{\partial \rho}$ is determined as:

$\frac{\partial c}{\partial \boldsymbol{\rho}}=\frac{\partial c}{\partial \check{\boldsymbol{\rho}}} \frac{\partial \check{\rho}}{\partial \boldsymbol{\rho}}$,

To calculate $\frac{\partial c}{\partial \check{\rho}}$, the adjoint approach described in the previous section is used:

$\frac{\partial c}{\partial \check{\boldsymbol{\rho}}_{l}}=\frac{\partial \tilde{c}}{\partial \check{\boldsymbol{\rho}}_{l}}=\left(\frac{\partial c}{\partial \check{\tilde{\boldsymbol{\rho}}}_{l}}+\boldsymbol{\lambda}_{l+1}^{T} \frac{\partial \breve{\mathbf{s}}_{l+1}}{\partial \check{\tilde{\boldsymbol{\rho}}}_{l}}\right) \frac{\partial \breve{\mathbf{s}}_{l}}{\partial \check{\boldsymbol{\rho}}_{l}}=\boldsymbol{\lambda}_{l}^{T} \frac{\partial \breve{\mathbf{s}}_{l}}{\partial \check{\boldsymbol{\rho}}_{l}}$,

where

$\boldsymbol{\lambda}_{l}^{T}=\frac{\partial c}{\partial \tilde{\check{\rho}}_{l}}+\boldsymbol{\lambda}_{l+1}^{T} \frac{\partial \breve{\mathbf{s}}_{l+1}}{\partial \check{\tilde{\rho}}_{l}} \quad$ for $1 \leq l \leq n_{k}$,

$\boldsymbol{\lambda}_{n_{k}}^{T}=\frac{\partial c}{\partial \check{\rho}_{n_{k}}}$

and $\frac{\partial c}{\partial \grave{\rho}}$ is calculated via direct differentiation using equation (30) and (31):

$\frac{\partial c}{\partial \stackrel{\circ}{\rho}}=\frac{\partial c}{\partial \hat{\hat{\hat{\rho}}}} \frac{\partial \hat{\hat{\hat{\rho}}}}{\partial \check{\hat{\hat{\rho}}}} \frac{\partial \hat{\hat{\tilde{\rho}}}}{\partial \hat{\hat{\rho}}} \frac{\partial \hat{\hat{\rho}}}{\partial \stackrel{\rho}{\rho}}$,

where $\frac{\partial c}{\partial \hat{\hat{\hat{\rho}}}}$ is calculated using the adjoint approach described in equation (25).

The sensitivity $\frac{\partial c}{\partial \check{\rho}}$ needed in equation (44) is obtained by concatenating the derivatives $\frac{\partial c}{\partial \check{\boldsymbol{\rho}}_{l}}$ for all individual layers. 